Supporting Information for

\title{
A quantitative model for reversibly photoswitchable sensors
}

Beatrice Adelizzi, ${ }^{* a}$ Vincent Gielen, ${ }^{b}$ Thomas Le Saux, ${ }^{a}$ Peter Dedecker, ${ }^{b}$ and Ludovic Jullien*a

${ }^{a}$ PASTEUR, Département de chimie, École normale supérieure, PSL University, Sorbonne Université, CNRS, 24, rue Lhomond, 75005 Paris, France; E-mail: beatrice.adelizzi@ens.psl.eu; ludovic.jullien@ens.psl.eu ${ }^{b}$ Laboratory for Nanobiology, Department of Chemistry, KU Leuven, Celestijnenlaan 200G, 3001 Heverlee, Belgium. 


\section{Contents}

1 Stepwise protocol for end-users 1

2 Experimental analysis on GCaMP6s-Q

$2.1 \quad$ UV-vis and fluorescence spectra $\ldots \ldots \ldots \ldots \ldots \ldots$

2.2 Estimation of the photoisomerization equilibrium constants and the brightness values of GCaMP6s-Q in its four fluorescent states . . . . . . . . . . . . . . 6

2.3 Fluorescence temporal evolution of GCaMP6s-Q photoisomerization: . . . . . . . . . . 8

2.4 GCaMP6s-Q- $\mathrm{Ca}^{2+}$ calibration under illumination $\ldots \ldots \ldots \ldots \ldots$

2.5 Application of GCaMP6s-Q in living cells . . . . . . . . . . . . . . . . . . 12

2.6 Calibration using the initial and final fluorescence values $\ldots \ldots \ldots \ldots$

2.7 Kinetics of change of the GCaMP6s-Q fluorescence signal $\ldots \ldots \ldots \ldots$

2.8 Light titration over a large range of light intensities $\ldots \ldots \ldots \ldots$

A Theoretical analysis of a reversibly photo-switchable sensor 17

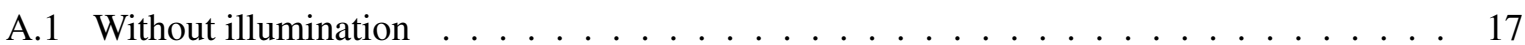

A.1.1 Thermodynamic aspects . . . . . . . . . . . . . . . . 17

A.1.2 Kinetic aspects . . . . . . . . . . . . . . . . 17

A.2 Under illumination $\ldots \ldots \ldots \ldots \ldots \ldots \ldots \ldots$

A.2.1 From the eight- to the four-state kinetic model $\ldots \ldots \ldots \ldots$

A.2.2 From the four- to two-state kinetic models . . . . . . . . . . . . . . . . . . . . . 19

B Light jump experiments $\quad 21$

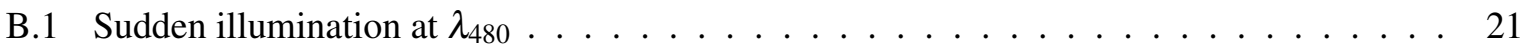

B.1.1 Rate-limiting photoisomerizations . . . . . . . . . . . . . . 21

B.1.2 Rate-limiting ligand exchanges . . . . . . . . . . . . . . . . . . . . . 24

B.2 Sudden change of illumination from $\lambda_{480}$ to $\lambda_{405} \ldots \ldots \ldots \ldots \ldots$

B.2.1 Rate-limiting photoisomerizations . . . . . . . . . . . . . 26

B.2.2 Rate-limiting ligand exchanges . . . . . . . . . . . . . . . . . . . . 27

$\begin{array}{ll}\text { References } & 30\end{array}$ 


\section{Stepwise protocol for end-users}

Based on the theoretical evaluation of rs-sensors (see Appendices A and B) and on the experimental results reported in the manuscript, we report a stepwise protocol to guide end-users in the utilization of rs-sensors. ${ }^{1}$

\section{Titrate the rs-sensor at $\mathbf{T}$ state using low light intensity}

- Measure fluorescence intensity of the rs-sensor at $\mathbf{T}$ state for each analyte concentration $[\mathbf{L}]$. Use [L] in the regime $0 \mathrm{nM}$ to $\approx 10 \cdot K_{0.5}$. An approximation of $K_{0.5}$ can be obtained by the information available on the sensing module.

- Plot the fluorescence intensity vs. the analyte concentration and fit with Hill equation to define $K_{0.5, T}$

- Fit Equation:

$$
\theta([\mathbf{L}])=\frac{[\mathbf{L}]^{v}}{[\mathbf{L}]^{v}+K_{0.5}^{v}}
$$

where $[\mathbf{L}]$ is the analyte concentration, $v$ is the Hill coefficient, and $K_{0.5}^{v}=K_{\mathrm{d}}$ is the dissociation constant of the sensor in its $\mathbf{T}$ state.

\section{Define illumination routine and tentative kinetic window}

- Suggested illumination routine is based on alternated square waves of lights at $\lambda_{1}$ and $\lambda_{2}$ (see Figure S1).

- Fix the initial kinetic window by relying on photochemical and kinetic information available from the rs-unit and sensing module in order to ensure that all the relaxation processes (photoswitching and analyte exchange) have enough time to occur under illumination.

- Note that the selected kinetic window is valid for the applied regime of light intensity as the thermodynamics and kinetics of photoisomerization depend on the light intensity. (See Eqs. 21, 22, 32, 33).

\section{Perform photoisomerization at asymptotic analyte concentrations}

- Perform photoisomerization at $[\mathbf{L}]=0 \mathrm{nM}$ and saturated conditions $[\mathbf{L}] \approx 10 \cdot K_{0.5}$ in the selected kinetic window. Repeat the experiment for 3-4 cycles at at least 4 different light intensities.

- Discard the first photoisomerization cycle at each light intensity.

- Perform a monoexponential fit on the other cycles and obtain the average of the relaxation time for saturated $\left.\left(<\tau_{\mathrm{TB} \rightarrow \mathrm{PB}, i}\right\rangle\right)$ and $\left.0 \mathrm{nM}\left(<\tau_{\mathrm{TU} \rightarrow \mathrm{PU}, i}\right\rangle\right)$ analyte concentration obtained for each light intensity (defined by its wavelength $\lambda_{i}$ and intensity $I_{i}$ ).

- Fit equation:

$$
F(t)=F(\text { end })+A_{\mathrm{T} \rightarrow \mathrm{P}, i} \cdot \exp \left(-\frac{t}{\tau_{\mathrm{T} \rightarrow \mathrm{P}, i}}\right)
$$

\footnotetext{
${ }^{1}$ Acronyms employed: $\mathbf{T}=$ thermodynamically stable state, $\mathbf{P}=$ photo-activated state, $\mathbf{B}=$ fully analyte-bound sensor, $\mathbf{U}=$ fully analyte-free sensor, $\mathbf{L}=$ analyte.
} 
where $t$ is the time in second and $A_{\mathrm{T} \rightarrow \mathrm{P}, i}$ is the amplitude of the fluorescence change. (See Eq. 35).

- Plot $\left\langle\tau_{\mathrm{TB} \rightarrow \mathrm{PB}, i}\right\rangle^{-1}$ and $\left\langle\tau_{\mathrm{TU} \rightarrow \mathrm{PU}, i}\right\rangle^{-1}$ vs. the light intensity applied $I_{i}$, perform a linear fit, and retrieve the photoisomerization cross sections for saturated and $0 \mathrm{nM}$ analyte concentration (i.e., $\sigma_{\mathrm{TB} \rightarrow \mathrm{PB}, i}$ and $\sigma_{\mathrm{TU} \rightarrow \mathrm{PU} i}$, respectively) from the slope of the linear fit.

- Fit equation:

$$
<\tau_{\mathrm{T} \rightarrow \mathrm{P}, i}>^{-1}\left(I_{i}\right)=k_{\mathrm{P} \rightarrow \mathrm{T}}^{\Delta}+\sigma_{\mathrm{T} \rightarrow \mathrm{P}, i} \cdot I_{i}
$$

- Fix the kinetic window for the next titration experiments.

- The kinetic window must be $\approx 5 \cdot \max \left(\tau_{\mathrm{TB} \rightarrow \mathrm{PB}, i}, \tau_{\mathrm{TU} \rightarrow \mathrm{PU} i}\right)$.

- Note that the selected kinetic window is valid for the light intensity regime applied in the photoisomerization experiment performed in the previous step. If the following experiment requires a different light intensity, a new kinetic window can be extracted by knowing the light intensity applied and the photoisomerization cross section. (See Eqs. 21, 22).

\section{Perform photoisomerization at intermediate analyte concentrations}

- Suggested experiment is performed by stepwise titration from $[\mathbf{L}]=0 \mathrm{nM}$ to $[\mathbf{L}]=10 \cdot K_{0.5}$

\section{Perform a fitting analysis on the fluorescence temporal evolution obtained for each analyte concentration under illumination}

- Fit equation: Eq. 2

\section{5 a) If the monoexponential fitting is satisfactory:}

- The monoexponential fit is good if the values of $\tau_{\mathrm{T} \rightarrow \mathrm{P}, i}$ obtained for each analyte concentration fall in the regime defined by the relaxation times obtained at asymptotic concentrations (i.e., $\tau_{\mathrm{TU} \rightarrow \mathrm{PU}, i}<\tau_{\mathrm{T} \rightarrow \mathrm{P}, i}<\tau_{\mathrm{TB} \rightarrow \mathrm{PB}, i}$ ) and fitting residues reflects random noise only.

- If the conditions are satisfied, the sensor is in the regime of fast analyte exchange and the photoisomerization is rate-limiting.

- To perform the calibration of the sensor, the user can exploit either the variation of $\tau_{\mathrm{T} \rightarrow \mathrm{P}, i}$ (see Eqs. 35, 66) or of the molecular brigthness $Q_{i}$ as a function of [L] (see Eq. 40). The experimental calibration curve so obtained can be fitted with Hill equation as defined in point 1 (Eq. 1).

5 b) If the monoexponential fitting is not satisfactory:

- The monoexponential fit does not describe well the trend if the values of $\tau_{\mathrm{T} \rightarrow \mathrm{P}, i}$ obtained for each analyte concentration fall out of the values obtained at asymptotic concentrations (i.e., $\tau_{\mathrm{T} \rightarrow \mathrm{P}, i}<\tau_{\mathrm{TU} \rightarrow \mathrm{PU}, i}$ and $\left.\tau_{\mathrm{T} \rightarrow \mathrm{P}, i}>\tau_{\mathrm{TB} \rightarrow \mathrm{PB}, i}\right)$, and/or the residues of the fit show a secondary trend.

- If the conditions above described are observed, the sensor is in the regime of slow analyte exchange and the ligand exchange is rate-limiting. 
- Perform a biexponential fit on the fluorescence temporal evolutions obtained for each analyte concentration.

- Fit equation:

$$
F(t)=F(\text { end })+A_{\mathrm{TB} \rightarrow \mathrm{PB}, i} \cdot \theta \cdot \exp \left(-\frac{t}{\tau_{\mathrm{TB} \rightarrow \mathrm{PB}, i}}\right)+A_{\mathrm{TU} \rightarrow \mathrm{PU}, i} \cdot(1-\theta) \cdot \exp \left(-\frac{t}{\tau_{\mathrm{TU} \rightarrow \mathrm{PU}, i}}\right)
$$

- Fix $A_{\mathrm{TB} \rightarrow \mathrm{PB}, i}$ and $\tau_{\mathrm{TB} \rightarrow \mathrm{PB}, i}$ equal to what obtained with the monoexpoential fit at $[\mathbf{L}]$ $=10 \cdot K_{0.5}$.

- Fix $A_{\mathrm{TU} \rightarrow \mathrm{PU}, i}$ and $\tau_{\mathrm{TU} \rightarrow \mathrm{PU}, i}$ equal to what obtained with the monoexpoential fit at $[\mathbf{L}]$ $=0 \mathrm{nM}$.

- Constrain $0 \leq \theta \leq 1$.

- To perform the calibration of the sensor, the user can exploit the variation of $\theta$ vs. [L] (see Eqs. 51,77). The experimental calibration curve so obtained can be fitted with Hill equation as defined in point $1 \mathrm{Eq} .1$.

- Additional Note: In the case that the rs-sensor does not show a significant change in brightness between its $\mathbf{T U}$ and its $\mathbf{P U}$ state at $[\mathbf{L}]=0 \mathrm{nM}$, the calibration can be performed with a monoexponential fit (See Eq. 55).

- Fit equation:

$$
F(t)=F(\text { end })+A_{\mathrm{TB} \rightarrow \mathrm{PB}, i} \cdot \theta \cdot \exp \left(-\frac{t}{\tau_{\mathrm{TB} \rightarrow \mathrm{PB}, i}}\right)
$$

- Fix $A_{\mathrm{TB} \rightarrow \mathrm{PB}}$ and $\tau_{\mathrm{TB} \rightarrow \mathrm{PB}, i}$ equal to what obtained with the monoexpoential fit at saturate analyte concentration.

- Constrain $0 \leq \theta \leq 1$. 

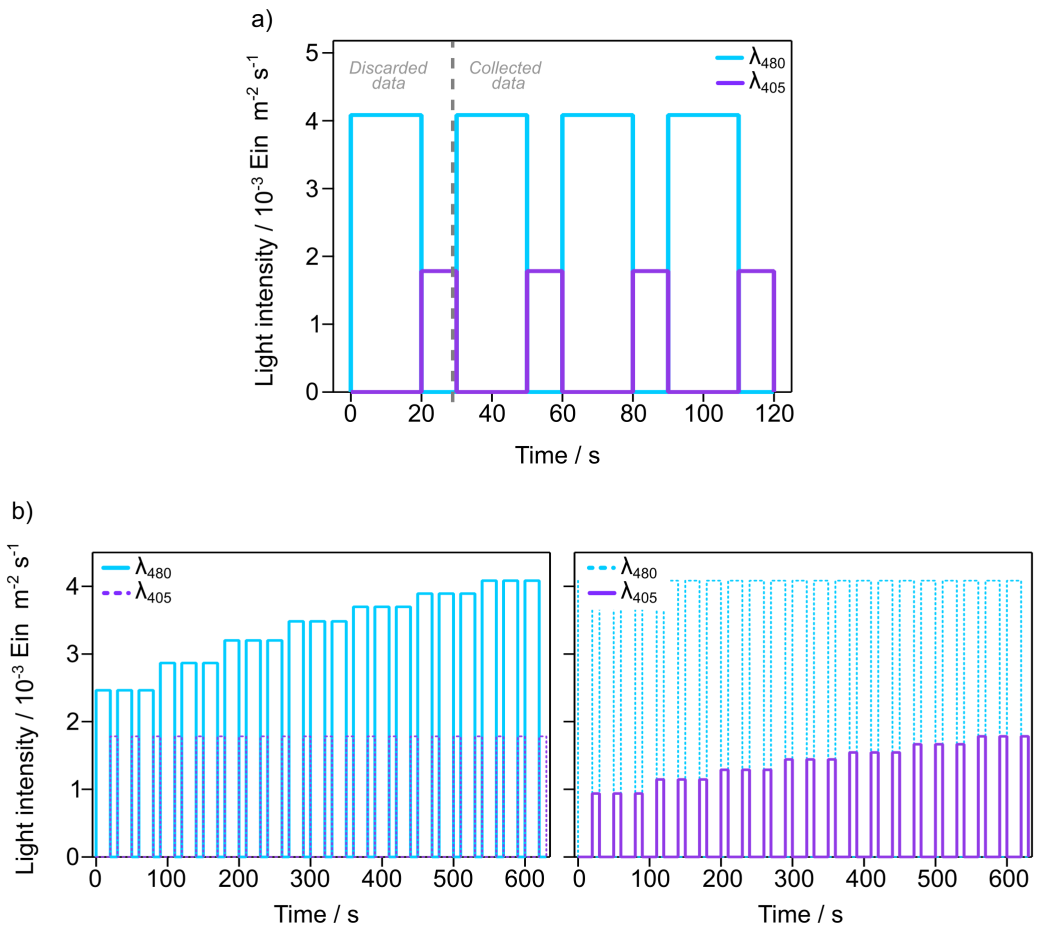

Figure S1: Light intensities applied for the photoisomerization studies by alternating square light waves at $\lambda_{480}$ (cyan line) and $\lambda_{405}$ (purple line) for a) calcium titration and b) light titration experiments. A similar routine is suggested for the application of the protocol above described. 


\section{Experimental analysis on GCaMP6s-Q}

\subsection{UV-vis and fluorescence spectra}

UV-vis spectra of GCaMP6s-Q in its analyte-bound and -free thermodynamically stable states, TB and $\mathbf{T U}$, were recorded at saturated calcium concentrations $\left(\left[\mathrm{Ca}^{2+}\right]_{\text {free }}=38.8 \mu \mathrm{M}\right)$ and in the absence of calcium $\left(\left[\mathrm{Ca}^{2+}\right]_{\text {free }}=0 \mathrm{nM}\right)$, respectively. The low intensity of illumination used during the measurements ensures the avoidance of photoisomerization. The large absorption red-shift associated to the $\mathrm{Ca}^{2+}$ binding (Table $\mathrm{S} 1$ ) indicates a change in the protonation state of the protein's chromophore (Figure S2a, dashed lines) and confirms that the calcium sensing module is allosterically coupled with the reversibly photoswitchable protein. Upon calcium binding, this coupling triggers a significant decrease of $\mathrm{pKa}\left(\mathrm{pKa}_{\mathrm{TB}}=6\right.$ and $\left.\mathrm{pKa}_{\mathrm{TU}}=10.2\right)$, and a concomitant rise in fluorescence intensity (Figure S2a, solid lines). ${ }^{2}$

At saturated calcium concentrations, and upon $480 \mathrm{~nm}$-illumination, GCaMP6s-Q is subjected to a photoisomerization reaction from its thermodynamically stable state to the photo-activated state (i.e., TB $\rightarrow$ PB). UV-vis and fluorescence spectra recorded at the initial (0) and final state (end) show that the photoisomerization reaction is associated to a change in the chromophore's protonation state and to a large decrease in fluorescence intensity (Figure S2b).

a)

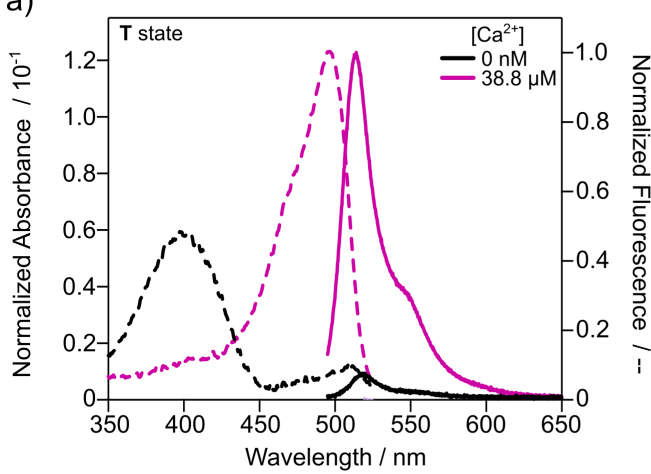

b)

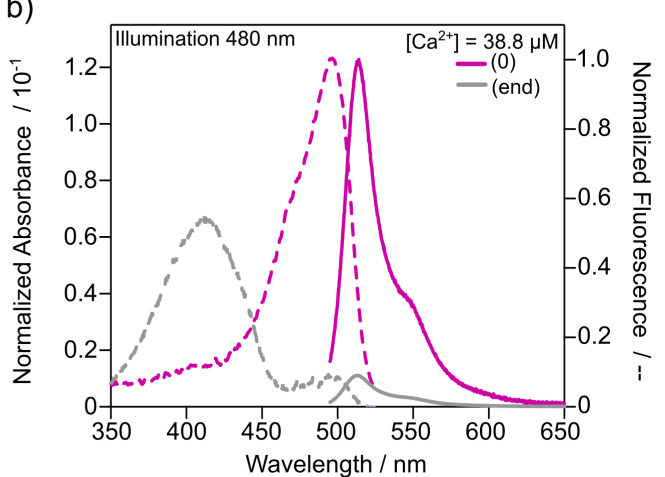

Figure S2: a) Normalized absorption (dashed lines) and emission (solid lines) spectra of GCaMP6s-Q in its TU state at $\left[\mathrm{Ca}^{2+}\right]_{\text {free }}=0$ (black lines) and in its $\mathbf{T B}$ state at $\left[\mathrm{Ca}^{2+}\right]_{\text {free }}=38.8 \mu \mathrm{M}$ (magenta lines). $\mathrm{b}$ ) Normalized absorption (dashed lines) and emission (solid lines) spectra of GCaMP6s-Q at $\left[\mathrm{Ca}^{2+}\right]_{\text {free }}=$ $38.8 \mu \mathrm{M}$ under $480 \mathrm{~nm}$ illumination at the initial state, (0), corresponding to TB state (magenta lines), and at the final state, (end), corresponding to $\mathbf{P B}$ state (gray lines). The absorption and emission spectra have been respectively normalized at $\lambda=278 \mathrm{~nm}$, and at $\lambda=515 \mathrm{~nm}$ using $F(0)_{480}$ at $\left[\mathrm{Ca}^{2+}\right]_{\text {free }}=38.8$ $\mu \mathrm{M}$ as reference.

Table S1: Photophysical values of GCaMP6s-Q

\begin{tabular}{c||cc} 
GCaMP6s-Q state & $\lambda_{\text {Abs }} / \mathrm{nm}$ & $\lambda_{\mathrm{Em}} / \mathrm{nm}$ \\
\hline TB & $(405), 496$ & 513 \\
TU & $400,(510)$ & 518 \\
PB & $412,(496)$ & 513 \\
PU & - & - \\
\hline
\end{tabular}

Absorption reported as: "main peak, (minor peak)" 


\subsection{Estimation of the photoisomerization equilibrium constants and the brightness values of GCaMP6s-Q in its four fluorescent states}

In the following, we aim to elucidate which information (brightness, photoswitching extent at steady-state) can be retrieved from applying the two-state model for processing experimental data obtained at different light intensity. In fact, the most attractive rs-sensors should exhibit maximal photoswitching extent and signal contrast between their photoswitched and non-photoswitched states.

As confirmed by the experiments reported in Figure 2 in the Main Text, the time response of the fluorescence signal of GCaMP6s-Q at asymptotic calcium concentration is reliably described by the two-state model. As detailed in Eqs. 32 and 42, this allows us to estimate the range of values of the constants reporting on the photoisomerization extent for the sensor in its bound $\left(\mathrm{K}_{\mathrm{TB} \rightarrow \mathrm{PB}}^{480}\right)$ and free $\left(\mathrm{K}_{\mathrm{TU} \rightarrow \mathrm{PU}}^{480}\right)$ states. The values so calculated are dependent on the applied light intensity and here are reported for the maximum light intensity applied in this work (i.e., $I_{480}=4.15 \cdot 10^{-3} \mathrm{Ein} \mathrm{m}^{-2} \mathrm{~s}^{-1}$ ).

The upper value of $\mathrm{K}_{\mathrm{TB} \rightarrow \mathrm{PB}}^{480}$ is extracted from Eq. 32 considering $\sigma_{\mathrm{PB} \rightarrow \mathrm{TB}, 480}=0$ and thus $\sigma_{\mathrm{TB} \rightarrow \mathrm{PB}, 480}+\sigma_{\mathrm{PB} \rightarrow \mathrm{TB}, 480}=\sigma_{\mathrm{TB} \rightarrow \mathrm{PB}, 480}$. Likewise, the upper value of $\mathrm{K}_{\mathrm{TU} \rightarrow \mathrm{PU}}^{480}$ is extracted from Eq. 32 considering $\sigma_{\mathrm{PU} \rightarrow \mathrm{TU}, 480}=0$ and thus $\sigma_{\mathrm{TU} \rightarrow \mathrm{PU}, 480}+\sigma_{\mathrm{PU} \rightarrow \mathrm{TU}, 480}=\sigma_{\mathrm{TU} \rightarrow \mathrm{PU}, 480}$. From the values of the sums of the cross sections extracted from Figure 2b,e Main Text and reported in Table S2, we retrieved $\operatorname{Max}\left(\mathrm{K}_{\mathrm{TB} \rightarrow \mathrm{PB}}^{480}\right)$ and $\operatorname{Max}\left(\mathrm{K}_{\mathrm{TU} \rightarrow \mathrm{PU}}^{480}\right)($ Table S3).

The lower value of $\mathrm{K}_{\mathrm{TB} \rightarrow \mathrm{PB}}^{480}$ is extracted from Eq. 42 from the experimental $R=3.55$ value by considering $Q_{\mathrm{PB}, 480}=0 \mathrm{M}^{-1} \mathrm{~cm}^{-1}$ (Table S3). Analogously, the lower value of $\mathrm{K}_{\mathrm{TU} \rightarrow \mathrm{PU}}^{480}$ is extracted from Eq. 42 by further considering the brightness of the unbound state to be always lower than that of the bound state as experimentally observed (Table S3).

Table S2: Fitting results of GCaMP6s-Q photoisomerization

\begin{tabular}{c||ccc||c}
\multicolumn{1}{c||}{$\left[\mathrm{Ca}^{2+}\right]_{\text {free }}$} & \multicolumn{3}{|c|}{$480 \mathrm{~nm}$ photoisomerization } & $405 \mathrm{~nm}$ photoisomerization \\
\hline \multirow{3}{*}{$38.8 \mu \mathrm{M}$} & $\sigma_{\mathrm{TB} \rightarrow \mathrm{PB}, 480}+\sigma_{\mathrm{PB} \rightarrow \mathrm{TB}, 480}$ & $\mathrm{k}_{\mathrm{PB} \rightarrow \mathrm{TB}}^{\Delta}$ & $\mathrm{m} A_{\mathrm{TB} \rightarrow \mathrm{PB}}{ }^{(\mathrm{a})}$ & $\sigma_{\mathrm{TB} \rightarrow \mathrm{PB}, 405}+\sigma_{\mathrm{PB} \rightarrow \mathrm{TB}, 405}$ \\
& {$\left[\mathrm{~m}^{2} \mathrm{~mol}^{-1}\right]$} & {$\left[\mathrm{s}^{-1}\right]$} & {$\left[\mathrm{Ein}^{-1} \mathrm{~m}^{2} \mathrm{~s}\right]$} & {$\left[\mathrm{mol}^{-1}\right]$} \\
& $70 \pm 1$ & $0.024 \pm 0.005$ & $143 \pm 1$ & $1097 \pm 74$ \\
\hline \multirow{3}{*}{$0 \mathrm{nM}$} & $\sigma_{\mathrm{TU} \rightarrow \mathrm{PU}, 480}+\sigma_{\mathrm{PU} \rightarrow \mathrm{TU}, 480}$ & $\mathrm{k}_{\mathrm{PU} \rightarrow \mathrm{TU}}^{\Delta}$ & $\mathrm{m} A_{\mathrm{TU} \rightarrow \mathrm{PU}}{ }^{(\mathrm{a})}$ & $\sigma_{\mathrm{TU} \rightarrow \mathrm{PU}, 405}+\sigma_{\mathrm{PU} \rightarrow \mathrm{TU}, 405}$ \\
& {$\left[\mathrm{~m}^{2} \mathrm{~mol}^{-1}\right]$} & {$\left[\mathrm{s}^{-1}\right]$} & {$\left[\mathrm{Ein}^{-1} \mathrm{~m}^{2} \mathrm{~s}\right]$} & {$\left[\mathrm{m}^{2} \mathrm{~mol}^{-1}\right]$} \\
& $21 \pm 2$ & $-0.012 \pm 0.007^{(\mathrm{b})}$ & $-79 \pm 6$ & $3667 \pm 224$ \\
\hline
\end{tabular}

(a) $m A_{\mathrm{TB} \rightarrow \mathrm{PB}}$ and $m A_{\mathrm{TU} \rightarrow \mathrm{PU}}$ are the slopes of the linearly fitted dependence of $A_{\mathrm{TB} \rightarrow \mathrm{PB}}$ and $A_{\mathrm{TU} \rightarrow \mathrm{PU}} v s$. $I_{480}$, respectively

(b) Since this value is physically impossible, we used $\mathrm{k}_{\mathrm{PB} \rightarrow \mathrm{TB}}^{\Delta}$ as an estimate for the calculation performed.

Table S3: Estimated GCaMP6s-Q photoisomerization constants and molecular brightnesses for $480 \mathrm{~nm}$

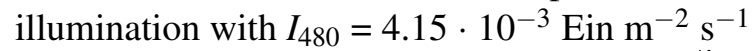

\begin{tabular}{c||cc|cc} 
Estimated range of $\mathrm{K}^{480} / \mathrm{nM}$ & \multicolumn{2}{|c|}{$\mathbf{T B} \rightarrow \mathbf{P B}$} & \multicolumn{2}{c}{$\mathbf{T U} \rightarrow \mathbf{P U}$} \\
& $2.55-11.96$ & \multicolumn{2}{c}{$0.134-3.59$} \\
\hline \multirow{2}{*}{ Estimated range of $Q_{480} / \mathrm{M}^{-1} \mathrm{~cm}^{-1}$} & $\mathbf{T B}$ & $\mathbf{P B}$ & $\mathbf{T U}$ & $\mathbf{P U}$ \\
& $19385^{(\mathrm{a})}$ & $0-4230^{(\mathrm{b})}$ & $1358^{(\mathrm{c})}$ & $1805-4230^{(\mathrm{b})}$ \\
\hline
\end{tabular}

(a) Calculated as reported in Eq. 41; (b) Calculated as reported in Eq. 42

$$
\text { (c) calculated as } \frac{F(0)_{\mathrm{TU}, 480}}{F(0)_{\mathrm{TB}, 480}} \cdot Q_{\mathrm{TB}, 480}
$$


The calculation above described (Table S3) can be adapted for each system that can be reliably described by the two-state model. However, for systems like GCaMP6s-Q a better estimation of the values of photoisomerization constants and brightnesses can be extracted by exploiting the linear dependence of the amplitude of the fluorescence change on the applied light intensity, whose slope $m A_{\mathrm{T} \rightarrow \mathrm{B}}$ is reported in Table S2. Such trend indicates that the quantity $K_{\mathrm{TB} \rightarrow \mathrm{PB}}^{480} /\left(1+K_{\mathrm{TB} \rightarrow \mathrm{PB}}^{480}\right)$ is independent on the light intensity (Eq. 38). Therefore, either $K_{\mathrm{TB} \rightarrow \mathrm{PB}}^{480}$ is light independent (i.e., $\sigma_{\mathrm{P} \rightarrow \mathrm{TB}, 480} \cdot I_{480} \gg k^{\Delta}$, Eq. 32) or $K_{\mathrm{TB} \rightarrow \mathrm{PB}}^{480} \gg 1$.

From $\operatorname{Min}\left(\mathrm{K}_{\mathrm{TB} \rightarrow \mathrm{PB}}^{480}\right)=2.55$ (i.e., $Q_{\mathrm{PB}, 480}=0 \mathrm{M}^{-1} \mathrm{~cm}^{-1}$ ), we deduce that $\sigma_{\mathrm{PB} \rightarrow \mathrm{TB}, 480} \cdot I_{480}$ is comparable with $k^{\Delta}$, which leads us to conclude that $\operatorname{Min}\left(K_{\mathrm{TB} \rightarrow \mathrm{PB}}^{480}\right)$ is light-dependent. Therefore, the observed linearity of the amplitude on the light intensity implies $K_{\mathrm{TB} \rightarrow \mathrm{PB}}^{480} \gg 1$ so as to consider $K_{\mathrm{TB} \rightarrow \mathrm{PB}}^{480} /\left(1+K_{\mathrm{TB} \rightarrow \mathrm{PB}}^{480}\right) \approx 1$. In this case Eq. 38 is simplified to Eq. 39 and from the slope of the linear fit, $m A_{\mathrm{TB} \rightarrow \mathrm{PB}, 480}$, (Table S2), we can retrieve an estimate of $Q_{\mathrm{PB}, 480}$ which is relevant over the whole range of light intensity investigated (Table $\mathrm{S} 4)$. Note also that $\operatorname{Max}\left(\mathrm{K}_{\mathrm{TB} \rightarrow \mathrm{PB}}^{480}\right)$ is intrinsically light dependent and the same conclusion can be drawn.

From $\operatorname{Min}\left(\mathrm{K}_{\mathrm{TU} \rightarrow \mathrm{PU}}^{480}\right)=0.134$ (i.e., $Q_{\mathrm{PU}, 480}=4230 \mathrm{M}^{-1} \mathrm{~cm}^{-1}$ ), we again deduce that $\sigma_{\mathrm{PU} \rightarrow \mathrm{TU}, 480} \cdot I_{480}$ is comparable with $k^{\Delta}\left(0.075\right.$ vs 0.024 , respectively), which leads us to conclude that $\operatorname{Min}\left(K_{\mathrm{TU} \rightarrow \mathrm{PU}}^{480}\right)$ is light-dependent. Therefore, also in this case, the observed linearity of the amplitude on the light intensity implies $K_{\mathrm{TU} \rightarrow \mathrm{PU}}^{480} \gg 1$ so to consider $K_{\mathrm{TU} \rightarrow \mathrm{PU}}^{480} /\left(1+K_{\mathrm{TU} \rightarrow \mathrm{PU}}^{480}\right) \approx 1$. In this case Eq. 38 is simplified to Eq. 39 and from the slope of the linear fit (Table S2), we can retrieve $Q_{\mathrm{PU}, 480}$ which is relevant over the whole range of light intensity investigated (Table S4). Note also that $\operatorname{Max}\left(\mathrm{K}_{\mathrm{TU} \rightarrow \mathrm{PU}}^{480}\right)$ is intrinsically light-dependent and the same conclusion can be drawn.

Table S4: Photoisomerization constants and GCaMP6s-Q brightnesses for $480 \mathrm{~nm}$ illumination accounting for the linear dependence of the amplitude of the fluorescence change on the light intensity.

\begin{tabular}{c||cc|cc} 
Estimated range of $\mathrm{K}^{480} / \mathrm{nM}$ & \multicolumn{2}{|c|}{$\mathbf{T B} \rightarrow \mathbf{P B}$} & \multicolumn{2}{|c}{$\mathbf{T U} \rightarrow \mathbf{P U}$} \\
& $2.55-11.96$ & \multicolumn{2}{c}{$>1$} \\
\hline \multirow{2}{*}{ Estimated range of $Q_{480} / \mathrm{M}^{-1} \mathrm{~cm}^{-1}$} & $\mathbf{T B}$ & $\mathbf{P B}$ & $\mathbf{T U}$ & $\mathbf{P U}$ \\
& 19385 & $7960^{(\mathrm{a})}$ & 1358 & $1925^{(\mathrm{b})}$ \\
\hline
\end{tabular}

(a) Calculated using $m A_{\mathrm{TB} \rightarrow \mathrm{PB}, 480}$ value reported in Table $\mathrm{S} 2$

(b) Calculated using $m A_{\mathrm{TU} \rightarrow \mathrm{BU}, 480}$ value reported in Table S2 


\subsection{Fluorescence temporal evolution of GCaMP6s-Q photoisomerization:}

Solutions of GCaMP6s-Q at intermediate $\left[\mathrm{Ca}^{2+}\right]_{\text {free }}$ were subjected to light jump experiment as previously described. Figure S3 shows the fluorescence temporal evolution of GCaMP6s-Q under 480 and $405 \mathrm{~nm}$ illumination as a function of the calcium concentration.
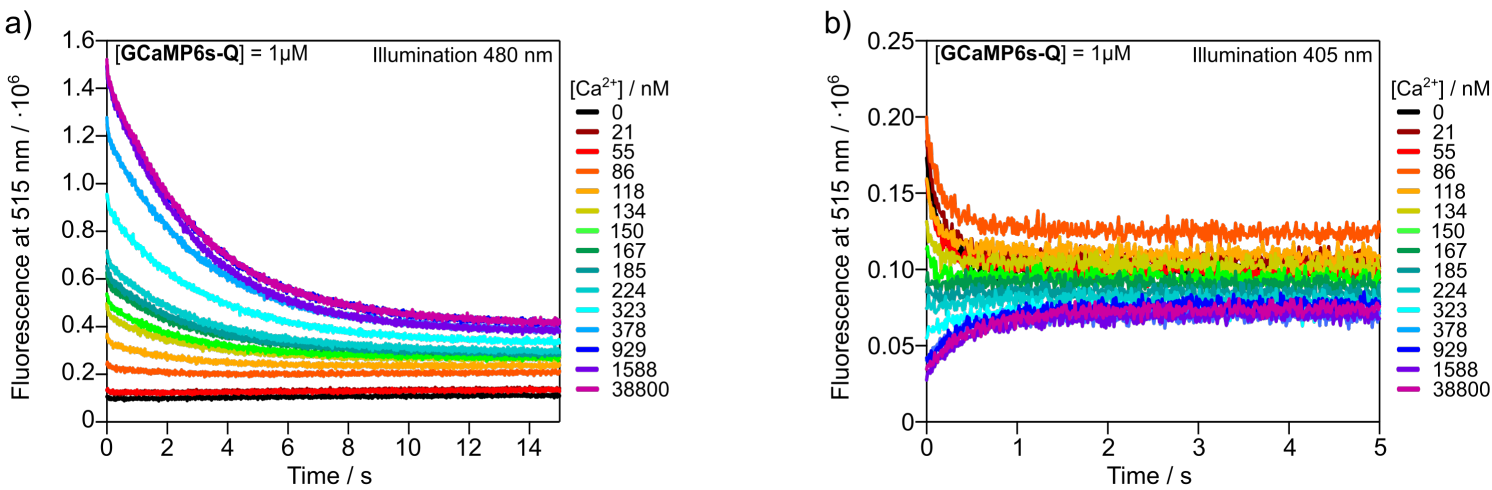

Figure S3: Second cycle of photoisomerization of GCaMP6s-Q followed as fluorescence temporal evolution at $515 \mathrm{~nm}$ under a) $480 \mathrm{~nm}$ and b) $405 \mathrm{~nm}$ illumination as a function of $\left[\mathrm{Ca}^{2+}\right]_{\text {free }}$. $\left[\right.$ GCaMP6s-Q] $=1 \mu \mathrm{M}$ in $30 \mathrm{mM}$ MOPS, $100 \mathrm{mM} \mathrm{KCl}$ and a buffered concentration of $\left[\mathrm{Ca}^{2+}\right]_{\text {free }}$ (CaEGTA/EGTA), $\mathrm{pH}=7.2,20^{\circ} \mathrm{C}$. Illumination intensity $I_{480}=4.15 \cdot 10^{-3} \mathrm{Ein} \mathrm{m}^{-2} \mathrm{~s}^{-1}$ and $I_{405}=1.78$

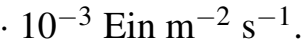

\subsection{GCaMP6s-Q-Ca ${ }^{2+}$ calibration under illumination}

Calibration under $480 \mathrm{~nm}$ illumination Based on Eqs. 4 35, we performed a fitting of all the recorded fluorescence temporal evolutions at each $\left[\mathrm{Ca}^{2+}\right]_{\text {free }}$ examined with the biexponential function given in Eq. 6 (Figure S4, blue lines).

$F(t)=F($ end $)+A_{\mathrm{TB} \rightarrow \mathrm{PB}, 480} \cdot \theta \cdot \exp \left(-t / \tau_{\mathrm{TB} \rightarrow \mathrm{PB}, 480}\right)+A_{\mathrm{TU} \rightarrow \mathrm{PU}, 480} \cdot(1-\theta) \cdot \exp \left(-t / \tau_{\mathrm{TU} \rightarrow \mathrm{PU}, 480}\right)$

By setting $A_{\mathrm{TB} \rightarrow \mathrm{PB}, 480}$ and $\tau_{\mathrm{TB} \rightarrow \mathrm{PB}, 480}$, and $A_{\mathrm{TU} \rightarrow \mathrm{PU}, 480}$ and $\tau_{\mathrm{TU} \rightarrow \mathrm{PU}, 480}$ as the amplitudes and relaxation times of the fully bound and free sensor extracted by fitting monoexponentially the fluorescence temporal evolution of GCaMP6s-Q $\mathbf{Q}$ at $\left[\mathrm{Ca}^{2+}\right]_{\text {free }}=38.8 \mu \mathrm{M}$ and $0 \mathrm{nM}$ under $480 \mathrm{~nm}$ illumination respectively, the fit returns the value of bound fraction $\theta$ for each $\left[\mathrm{Ca}^{2+}\right]_{\text {free }}$ examined.

On the same set of data, a simplified fit was performed (Figure S4a, black lines) applying Eq. 7 from considering that $Q_{\mathrm{TU}, 480} \approx Q_{\mathrm{PU}, 480}$ (see Table S4) as reported in Eqs. 555

$$
F(t)=F(\text { end })+A_{\mathrm{TB} \rightarrow \mathrm{PB}, 480} \cdot \theta \cdot \exp \left(-t / \tau_{\mathrm{TB} \rightarrow \mathrm{PB}, 480}\right)
$$

By setting $A_{\mathrm{TB} \rightarrow \mathrm{PB}, 480}$ and $\tau_{\mathrm{TB} \rightarrow \mathrm{PB}, 480}$ as the amplitude and relaxation time of the fully bound sensor extracted by fitting monoexponentially the fluorescence temporal evolution of GCaMP6s-Q at $\left[\mathrm{Ca}^{2+}\right]_{\text {free }}$ $=38.8 \mu \mathrm{M}$ under $480 \mathrm{~nm}$ illumination, the fit again returns the value of bound fraction $\theta$ for each $\left[\mathrm{Ca}^{2+}\right]_{\text {free }}$ examined.

The biexponential fit well describes the system (Figure S4, blue lines) at each $\left[\mathrm{Ca}^{2+}\right]_{\text {free. }}$ Moreover, due to the low amplitude of the fluorescence time response of the ligand-free sensor compared to that of the 
ligand-bound, the overall monoexponential fitting describes with satisfactory accuracy the GCaMP6s-Q fluorescence time response (Figure S4, black lines). In fact, the monoexponential fit does not describe well the fluorescence time response only at low calcium concentration, as expected. This conclusion is further supported by the comparison between the Hill calibration curve obtained via the monoexponential and that extracted by the biexponential fit (Figure S4b). The monoexponential fit may be relevant to facilitate calibration for rs-sensors that show large difference in the amplitude of photoisomerization between their ligand-bound and ligand-free state. This simplifies the treatment allowing the direct use of the extracted amplitude for calibrating the system as long as the sensor concentration and the light intensity is kept constant between the sensors calibration and its exploitation.

Calibration under $405 \mathrm{~nm}$ illumination Analogous treatment was performed for the time response of the fluorescence signal under $405 \mathrm{~nm}$ illumination (Figure S5a blue curves).Biexponential fit based on Eq. 77 was performed applying Eq. 8

$F(t)=F($ end $)+A_{\mathrm{TB} \rightarrow \mathrm{PB}, 405} \cdot \theta_{480} \cdot \exp \left(-t / \tau_{\mathrm{TB} \rightarrow \mathrm{PB}, 405}\right)+A_{\mathrm{TU} \rightarrow \mathrm{PU}, 405} \cdot\left(1-\theta_{480}\right) \cdot \exp \left(-t / \tau_{\mathrm{TU} \rightarrow \mathrm{PU}, 405}\right)$

By setting $A_{\mathrm{TB} \rightarrow \mathrm{PB}, 405}$ and $\tau_{\mathrm{TB} \rightarrow \mathrm{PB}, 405}$, and $A_{\mathrm{TU} \rightarrow \mathrm{PU}, 405}$ and $\tau_{\mathrm{TU} \rightarrow \mathrm{PU}, 405}$ as the amplitudes and relaxation times of the fully bound and ligand-free sensor states extracted by fitting monoexponentially the fluorescence temporal evolutions of GCaMP6s-Q at $\left[\mathrm{Ca}^{2+}\right]_{\text {free }}=38.8 \mu \mathrm{M}$ and $0 \mathrm{nM}$ under $405 \mathrm{~nm}$ illumination, the fit returns the value of bound fraction $\theta_{480}$ for each $\left[\mathrm{Ca}^{2+}\right]_{\text {free }}$ examined. Since $A_{\mathrm{TB} \rightarrow \mathrm{PB}, 405}$ and $A_{\mathrm{TU} \rightarrow \mathrm{PU}, 405}$ are comparable, the simplification to the monoexponential fit (as the one presented for $480 \mathrm{~nm}$-driven photoisomerization) cannot be reliably used in the case of $405 \mathrm{~nm}$-driven photoisomerization to calibrate the system (not reported). 
a)
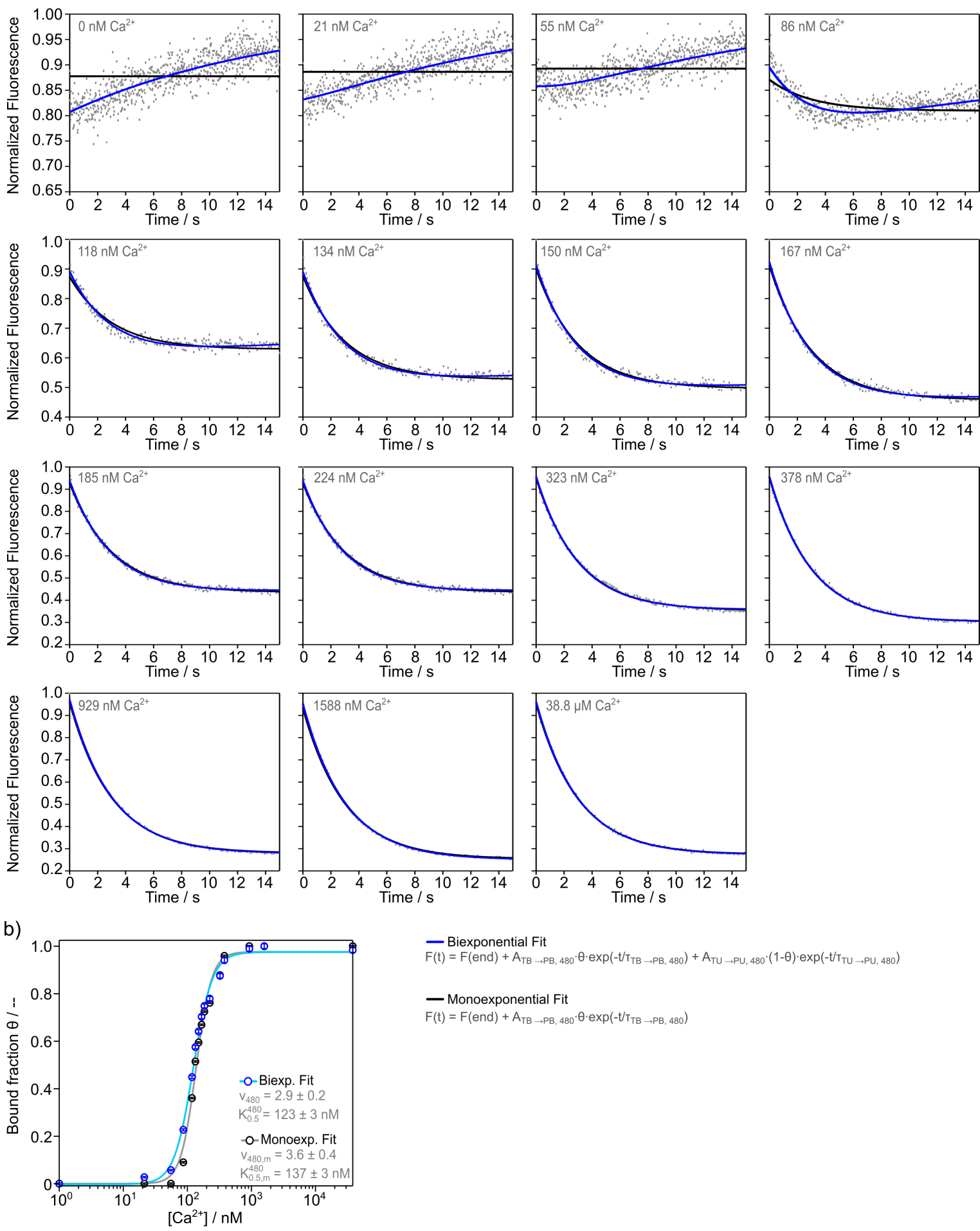

- Biexponential Fit

$F(t)=F($ end $)+A_{T B} \rightarrow P B, 480 \cdot \theta \cdot \exp \left(-t / T_{T B} \rightarrow P B, 480\right)+A_{T U} \rightarrow P U, 480^{\circ} \cdot(1-\theta) \cdot \exp \left(-t / T_{T U} \rightarrow P U, 480\right)$

- Monoexponential Fit

$F(t)=F(e n d)+A_{T B} \rightarrow P B, 480 \cdot \theta \cdot \exp \left(-t / T_{T B} \rightarrow P B, 480\right)$

Figure S4: a) GCaMP6s-Q fluorescence temporal evolution under $480 \mathrm{~nm}$ illumination (gray dots) at $\left[\mathrm{Ca}^{2+}\right]_{\text {free }}$ from $0 \mathrm{nM}$ (top left) to $1.588 \mu \mathrm{M}$ (bottom right). Fitting performed with either a biexponential equation (blue lines, held parameters: $A_{\mathrm{TB} \rightarrow \mathrm{PB}, 480}, \tau_{\mathrm{TB} \rightarrow \mathrm{PB}, 480}, A_{\mathrm{TU} \rightarrow \mathrm{PU}, 480}$, and $\tau_{\mathrm{TU} \rightarrow \mathrm{PU}, 480}$ ) or with a monoexponential equation (black lines, held parameters: $A_{\mathrm{TB} \rightarrow \mathrm{PB}, 480}$ and $\tau_{\mathrm{TB} \rightarrow \mathrm{PB}, 480}$ ). b) Non linear regression of bound fraction estimated from biexponential (blue dots) or monoexponential (black dots) fits on the temporal evolution of fluo emission. Each regression was fitted with Hill equation to obtain the related thermodynamic parameters (cyan and gray lines, respectively). Measurements reported for the second photoisomerization cycle with [GCaMP6s-Q] $=1 \mu \mathrm{M}$ in $30 \mathrm{mM}$ MOPS, $100 \mathrm{mM} \mathrm{KCl}$ and a buffered concentration of $\left[\mathrm{Ca}^{2+}\right]_{\text {free }}(\mathrm{CaEGTA} / \mathrm{EGTA}), \mathrm{pH}=7.2,20{ }^{\circ} \mathrm{C}$. Illumination intensity $I_{480}=$ $4.15 \cdot 10^{-3}$ Ein $\mathrm{m}^{-2} \mathrm{~s}^{-1}$. 

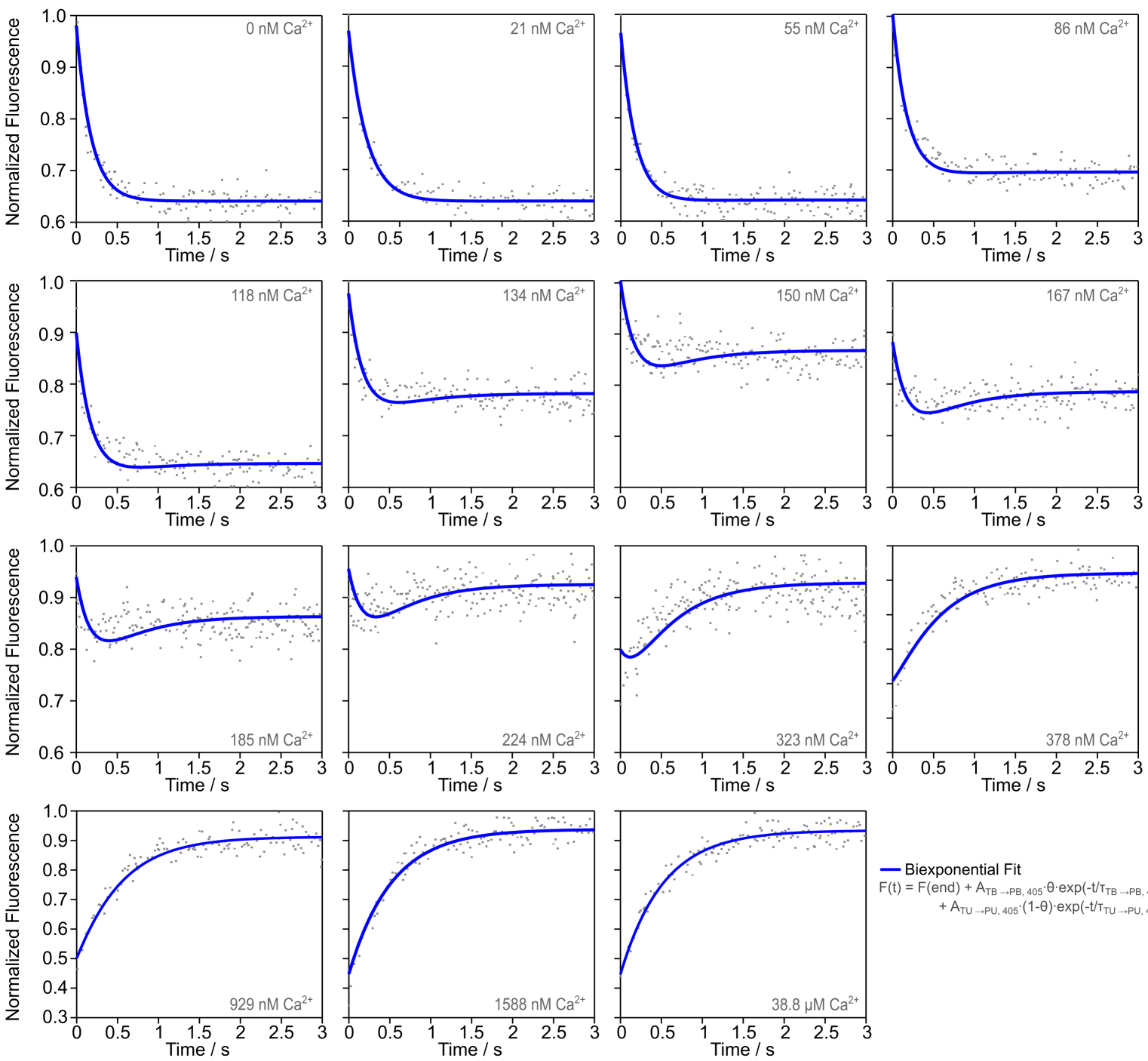

- Biexponential Fit

$F(t)=F($ end $)+A_{T B} \rightarrow P B, 405 \cdot \theta \cdot \exp \left(-t / T_{T B} \rightarrow P B, 480\right)$ $+\mathrm{A}_{\mathrm{TU} \rightarrow \mathrm{PU}, 405^{5} \cdot(1-\theta) \cdot \exp \left(-\mathrm{t} / \mathrm{T}_{\mathrm{T} U} \rightarrow \mathrm{PU}, 480\right)}$

Figure S5: Fluorescence temporal evolution under $405 \mathrm{~nm}$ illumination for GCaMp6s-Q (gray dots) at intermediate calcium concentrations from $\left[\mathrm{Ca}^{2+}\right]_{\text {free }}=0 \mathrm{nM}$ (top left) to $\left[\mathrm{Ca}^{2+}\right]_{\text {free }}=38.8 \mu \mathrm{M}$ (bottom right). Fitting performed with a biexponential equation (blue lines, parameters, $A_{\mathrm{TB} \rightarrow \mathrm{PB}, 405}$, $\tau_{\mathrm{TB} \rightarrow \mathrm{PB}, 405}, A_{\mathrm{TU} \rightarrow \mathrm{PU}, 405}$, and $\tau_{\mathrm{TU} \rightarrow \mathrm{PU}, 405}$ maintained held). Measurements reported for the second photoisomerization cycle with [GCaMP6s-Q] $=1 \mu \mathrm{M}$ in $30 \mathrm{mM}$ MOPS, $100 \mathrm{mM} \mathrm{KCl}$ and a buffered concentration of $\left[\mathrm{Ca}^{2+}\right]_{\text {free }}(\mathrm{CaEGTA} / \mathrm{EGTA}), \mathrm{pH}=7.2,20^{\circ} \mathrm{C}$. Illumination intensity $I_{405}=1.78 \cdot 10^{-3}$ Ein $\mathrm{m}^{-2} \mathrm{~s}^{-1}$. 


\subsection{Application of GCaMP6s-Q in living cells}

To further evaluate the reliability of the method proposed, photoisomerization of cell-expressed GCaMP6s-Q as a function of $\left[\mathrm{Ca}^{2+}\right]_{\text {free }}$ was tested by retreating the data reported by Gielen et al. ${ }^{2}$ in living Hela cells. For each cell, the temporal evolution of the normalized fluorescence signal under $480 \mathrm{~nm}$ illumination was analyzed following the protocol used for in vitro experiments (see sections 1 , $2.3,2.4)$. On a set of 10 cells exposed to $480 \mathrm{~nm}$-driven photoisomerization under saturated conditions $\left(\left[\mathrm{Ca}^{2+}\right]_{\text {free }}=39 \mu \mathrm{M}\right)$, monoexponential fits gave consistent values of $\tau_{\mathrm{TB} \rightarrow \mathrm{PB}, 480}$ with an average $\left\langle\tau_{\mathrm{TB} \rightarrow \mathrm{PB}, 480}>=0.15 \mathrm{~s} \pm 0.01\right.$. Based on the cross section calculated in vitro, the light intensity applied at the sample level is $I_{480}=0.09 \mathrm{Ein} \mathrm{m}^{-2} \mathrm{~s}^{-1}$. This value falls in the regime of linearity $\tau_{\mathrm{TB} \rightarrow \mathrm{PB}, 480}^{-1} \mathrm{vs}$. $I_{480}$ as described in Figure S9.

Calcium titration was then performed on each cell subjected to $480 \mathrm{~nm}$-driven photoisomerization by applying a monoexponential fit on the fluorescence temporal evolutions recorded at each $\left[\mathrm{Ca}^{2+}\right]_{\text {free }}$ concentration. As reported for the analysis in vitro (Eq. 7), a monoexponential fit was applied to retrieve the bound fraction $\theta$ by fixing $\tau=\tau_{\mathrm{TB} \rightarrow \mathrm{PB}, 480}$ and $A=A_{\mathrm{TB} \rightarrow \mathrm{PB}, 480}$ (Figure S6a). The resulting distribution of 10 titration curves compares well with the titration curve obtained in cuvette with homogeneous solutions (Figure S6b). More generally, these experiments confirm that our method can reliably be used under epifluorescence microscope conditions and on living cells.

a)

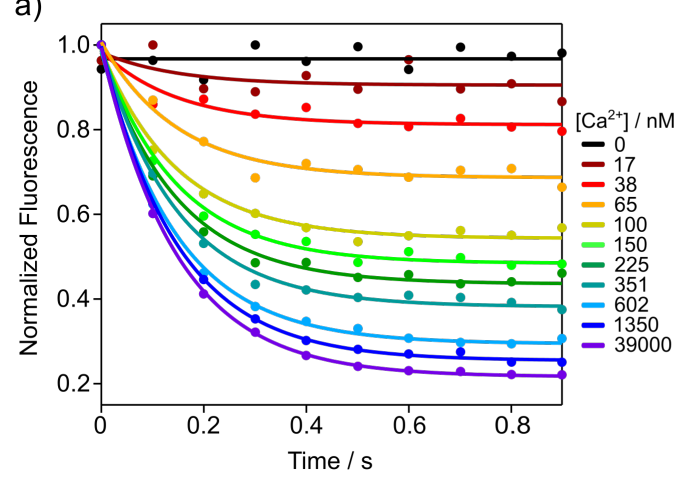

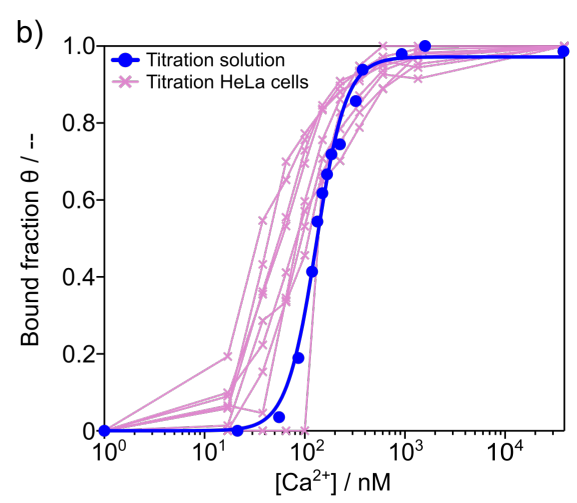

Figure S6: a) Temporal evolution of the normalized fluorescence response of GCaMP6s-Q expressed in a single HeLa cell to $480 \mathrm{~nm}$ illumination (the fluorescence signal was recorded at $515 \mathrm{~nm}$ and normalized by the highest value at each $\left[\mathrm{Ca}^{2+}\right]_{\text {free }}$ tested). The experimental points (markers) were fitted with a monoexponential function (lines) to retrieve the bound fraction $\theta$ from the respective amplitudes of the exponential terms upon fixing $\tau_{\mathrm{TB} \rightarrow \mathrm{PB}, 480}$ and $A_{\mathrm{TB} \rightarrow \mathrm{PB}, 480}$ corresponding to the fully-bound state reached at saturated condition. b) Dependence of $\theta$ on $\left[\mathrm{Ca}^{2+}\right]_{\text {free }}$ as extracted from the monoexponenital fit of the temporal evolution of the fluorescence values under $480 \mathrm{~nm}$ illumination. Titration in vivo performed on a set of living cells $\left(I_{480}=9.510^{-2}\right.$ Ein $\left.\mathrm{m}^{-2} \mathrm{~s}^{-1}\right)$ is reported (rose lines and markers) and compared with the calibration curve obtained in vitro $\left(I_{480}=4.1510^{-3} \mathrm{Ein} \mathrm{m}^{-2} \mathrm{~s}^{-1}\right)$ with the monoexponential fitting approximation (blue dots, fitted with an Hill fitting curve). 


\subsection{Calibration using the initial and final fluorescence values}

Under the assumption that the fluorescence change associated to calcium exchange is the rate limiting step governing the GCaMP6s-Q signal, the bound fractions obtained by fitting the time evolution of the GCaMP6s-Q fluorescence as a function of $\left[\mathrm{Ca}^{2+}\right]_{\text {free }}$ under $480 \mathrm{~nm}$ illumination must coincide with the bound fraction calculated for GCaMP6s-Q extracted from analyzing the initial signal under $480 \mathrm{~nm}$ illumination, which yields titration in the $\mathbf{T}$ state. Likewise, the bound fractions obtained by fitting the time evolution of the GCaMP6s-Q fluorescence as a function of $\left[\mathrm{Ca}^{2+}\right]_{\text {free }}$ under 405 $\mathrm{nm}$ illumination must coincide with the bound fraction extracted from analyzing the final signal in the regime of intermediate times under $480 \mathrm{~nm}$ illumination (considering that we found $\mathrm{K}_{\mathrm{T} \rightarrow \mathrm{P}, 480}^{480} \gg 1$ (See appendices A, B), this signal essentially arises from GCaMP6s-Q in its $\mathbf{P}$ state).

The bound fractions of GCaMP6s-Q (Figure 3c bottom, Main Text) were extracted from the initial and final fluorescence values recorded over the investigated kinetic window under $480 \mathrm{~nm}$ illumination (Figure S7). The evolution of $F_{480}(0) v s . \quad\left[\mathrm{Ca}^{2+}\right]_{\text {free }}$ can be reliably fitted by Hill equation, which yields the thermodynamic values of calcium binding of the GCaMP6s- $Q \mathbf{T}$ state $\left(\mathrm{K}_{0.5, \mathrm{~T}, 480}\right.$ and $\left.v_{\mathrm{T}, 480}\right)$. Analogously, from the evolution of $F_{480}$ (end $)$ vs. $\left[\mathrm{Ca}^{2+}\right]_{\text {free }}$, the thermodynamic values of calcium binding of the GCaMP6s-Q $\mathbf{P}$ state $\left(\mathrm{K}_{0.5, \mathrm{PT}, 480}\right.$ and $v_{\mathrm{P}, 480}$ are retrieved based on considering that $\left.\mathrm{K}_{\mathrm{T} \rightarrow \mathrm{P}, 480}^{480} \gg 1\right)$.

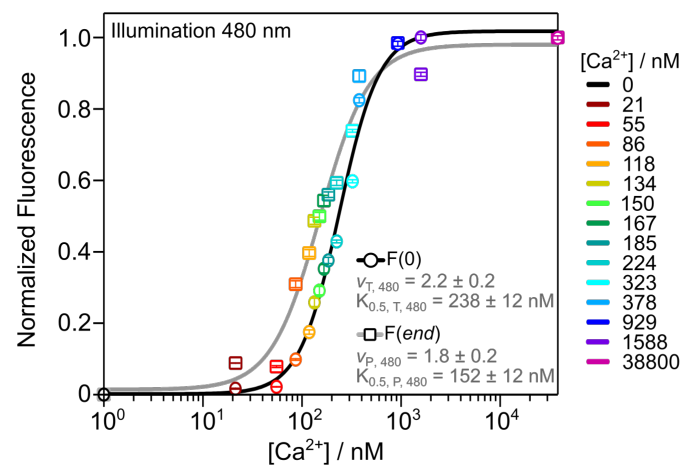

Figure S7: Dependence of GCaMP6s-Q normalized initial (circles, black line) and final (squares, gray line) fluorescence on $\left[\mathrm{Ca}^{2+}\right]_{\text {free }}$ upon $480 \mathrm{~nm}$ illumination $\left(I_{480}=4.15 \cdot 10^{-3} \mathrm{Ein} \mathrm{m}^{-2} \mathrm{~s}^{-1}\right)$. Fluorescence values calculated as average \pm sd over three consecutive photocycles. 


\subsection{Kinetics of change of the GCaMP6s-Q fluorescence signal}

In non-photoisomerizable GCaMP sensors, $\mathrm{Ca}^{2+}$ binding yields a cascade of conformational changes involving multiple intermediate states with their own brightness. ${ }^{1,3,5,6}$ Cumulatively, two main steps can be observed by following the temporal evolution of the fluorescence signal. The first process consists in the binding of calcium ions to the CaM unit. It is very fast in the order of tens of ms. The second process includes the interaction of $\mathrm{Ca}^{2+}-\mathrm{CaM}$ with the RS20 peptide. Involving the reorganizations of the sensor folding, its relaxation time can reach up to 1 second for the slowest sensors. ${ }^{1,3,5,6}$ Such a time scale is similar to the one of GCaMP6s-Q photoisomerization in our experiments and motivates the assumption that the calcium-driven fluorescence change is rate-limiting with respect to the photoisomerization-driven one. This assumption accounts for the observed biexponential decay of the fluorescence signal which is interpreted as a sum of the photoisomerization-driven fluorescence changes in the calcium-bound and calcium-free sensor states (Eqs. 6, 8). For evaluating this assumption, we performed stopped-flow experiments to investigate the kinetics of fluorescence change driven by calcium binding in GCaMP6s-Q. Although dealing with GCaMP6s-Q in its ground state instead of its photo-activated one (as encountered in our illumination experiments), we estimated that these experiments could provide interesting hints.

First, we addressed the calcium on-rate by investigating the fluorescence change associated to the sensor complexation by mixing GCaMP6s-Q at $\left[\mathrm{Ca}^{2+}\right]=0 \mathrm{nM}$ with a solution of $10 \mathrm{mM} \mathrm{CaCl}_{2}$ in PBS (pH = 7.4). Upon mixing, the final concentration of calcium is $\left[\mathrm{Ca}^{2+}\right]_{\text {free }}=5 \mu \mathrm{M}$. By stopped-flow we observed a very fast rise of the fluorescence signal at a time scale falling within, or very closely, to the instrumental dead-time $\left(\tau_{\text {on-sat }}<30 \mathrm{~ms}\right.$ ) (Figure S8a). This result is in line with previously reported behavior for GCaMP3 and GCaMP6 mutants. ${ }^{1,3,5,6}$

In a second experiment, we addressed the off-rate by following the fluorescence change associated to sensor decomplexation by mixing GCaMP6s-Q under saturated calcium conditions ([GCaMP6s-Q] = $0.5 \mu \mathrm{M}$ in aqueous solution containing $1 \mathrm{mM} \mathrm{CaCl}_{2}$ in PBS, $\mathrm{pH}=7.4$ ) with a $10 \mathrm{mM}$ EGTA solution in PBS ( $\mathrm{pH}=7.4)$ in order to get a vanishing final buffered concentration $\left[\mathrm{Ca}^{2+}\right]_{\text {free. }}$ The drop of the fluorescence signal was monoexponentially fitted to retrieve a relaxation time $\tau_{\text {off }}=1.6 \mathrm{~s}$ (Figure S8b). In line with previously reported observations for GCaMP mutants, ${ }^{1,3,5,6}$ this value is lower than the relaxation times, which have been extracted from the monoexponential fits on the calcium-bound sensor state at the light intensities applied in this study. Overall both experiments have supported the relevance of a photoisomerization rate-limiting two-state model to account for the fluorescence change occurring upon illumination of the calcium-bound sensor.

Then we addressed the biexponential trend of the fluorescence change observed under illumination at intermediate calcium concentrations. We mixed GCaMP6s-Q at $\left[\mathrm{Ca}^{2+}\right]_{\text {free }}=0 \mathrm{nM}$ with a buffered solution at $\left[\mathrm{Ca}^{2+}\right]_{\text {free }}=39 \mu \mathrm{M}$ resulting in a final buffered $\left[\mathrm{Ca}^{2+}\right]_{\text {free }}=151 \mathrm{nM}$. In this condition, the fluorescence rise is much slower than at high calcium concentrations. It occurs at a time scale $\left(\tau_{\mathrm{on}}=8 \mathrm{~s}\right)$ (Figure S8c), which is now comparable with the one observed during the photoisomerization $\left(\tau_{\mathrm{TU} \rightarrow \mathrm{PU}, 480}\right.$ $=15 \mathrm{~s}$ ) under similar conditions of calcium concentration (Figure 2a bottom Main Text). Therefore this experiment has supported the relevance of a two-state model involving rate-limiting calcium-induced conformational changes to account for the fluorescence change occurring under illumination of the calcium-bound sensor. 

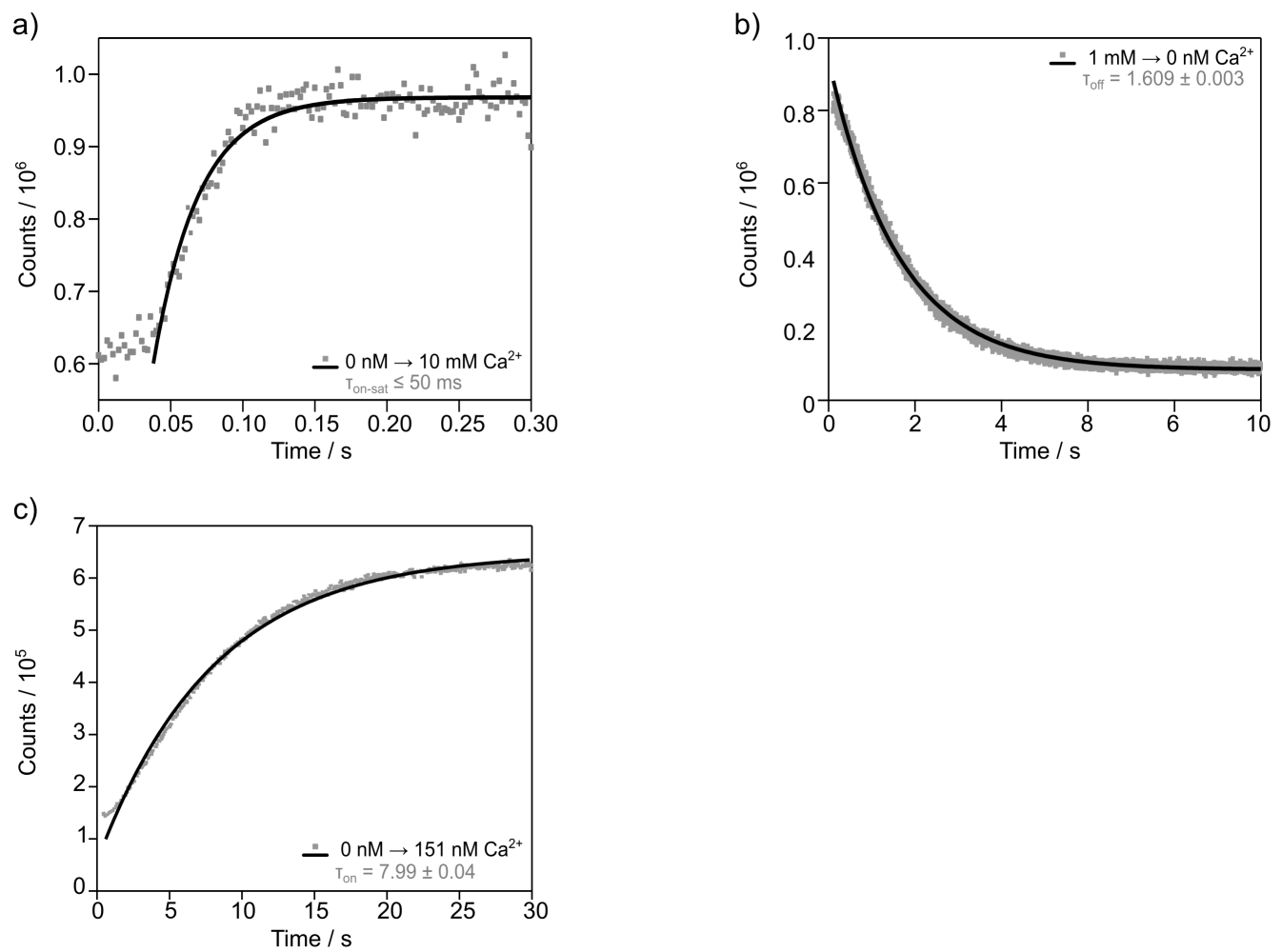

Figure S8: Stopped flow investigation of the kinetics of the GCaMP6s-Q fluorescence changes resulting from changes of the calcium concentration. a) Fluorescence temporal evolution upon sudden 1:1 mixing of $0.5 \mu$ M GCaMP6s-Q at $\left[\mathrm{Ca}^{2+}\right]_{\text {free }}=0 \mathrm{nM}$ with $10 \mathrm{mM} \mathrm{CaCl}_{2}$ in PBS, finally resulting in $\left[\mathrm{Ca}^{2+}\right]_{\text {free }}$ $=5 \mathrm{mM}$. The kinetics observed fall within the instrumental dead-time; b) Fluorescence temporal evolution upon sudden mixing of $0.5 \mu \mathrm{M}$ GCaMP6s-Q in $1 \mathrm{mM} \mathrm{CaCl}_{2}$ PBS with $10 \mathrm{mM}$ EGTA in PBS, finally resulting in $\left[\mathrm{Ca}^{2+}\right]_{\text {free }}=0 \mathrm{nM}$; c) Fluorescence temporal evolution upon sudden $1: 1$ mixing of $0.5 \mu \mathrm{M}$ GCaMP6s-Q at $\left[\mathrm{Ca}^{2+}\right]_{\text {free }}=0 \mathrm{nM}$ with a buffered solution of $\left[\mathrm{Ca}^{2+}\right]_{\text {free }}=39 \mu \mathrm{M}$, finally resulting in $\left[\mathrm{Ca}^{2+}\right]_{\text {free }}=151 \mathrm{nM}$. Each relaxation time is calculated as average $\pm \mathrm{sd}(\mathrm{n}=4-7)$. Excitation source, LED $I_{480}=8.3 \cdot 10^{-6}$ Ein $\mathrm{m}^{-2} \mathrm{~s}^{-1}$. 


\subsection{Light titration over a large range of light intensities}

The linear dependence of $\tau_{\mathrm{TB} \rightarrow \mathrm{PB}, 480}$ vs. $I_{480}$ (Main Text Figure 2) over a larger range of light intensity was evaluated by analyzing the fluorescence temporal response of GCaMP6s-Q under epifluorescence microscope conditions. The photoisomerization of GCaMP6s-Q was recorded in vitro on a home-built epifluorescence microscope. The experiment was performed at $20^{\circ} \mathrm{C}$ using $480 \mathrm{~nm}$ LED in a sandwiched coverslip chamber (thickness $80 \mu \mathrm{M}$ ). GCaMP6s-Q was dissolved in a buffer solution with composition identical to what employed for the cuvette experiments.

In line with what recorded in the fluorimeter, the $480 \mathrm{~nm}$-driven photoisomerization $\left(I_{480}=0.138\right.$ Ein $\left.\mathrm{m}^{-2} \mathrm{~s}^{-1}\right)$ at saturated calcium concentrations $\left(\left[\mathrm{Ca}^{2+}\right]_{\text {free }}=38.8 \mu \mathrm{M}\right)$ shows GCaMP6s-Q fluorescence exponentially decaying over time. The monoexponential fit of the recorded fluorescence temporal evolution yields $\tau_{\mathrm{TB} \rightarrow \mathrm{PB}, 480}=0.07 \mathrm{~s}$ (Figure S9a). This well aligns with the calibration obtained in

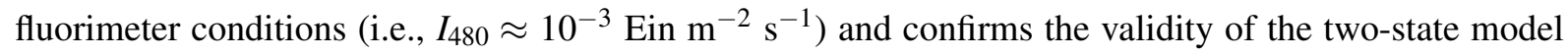
under epifluorescence conditions (Figure 2 Main Text and Figure S9b).
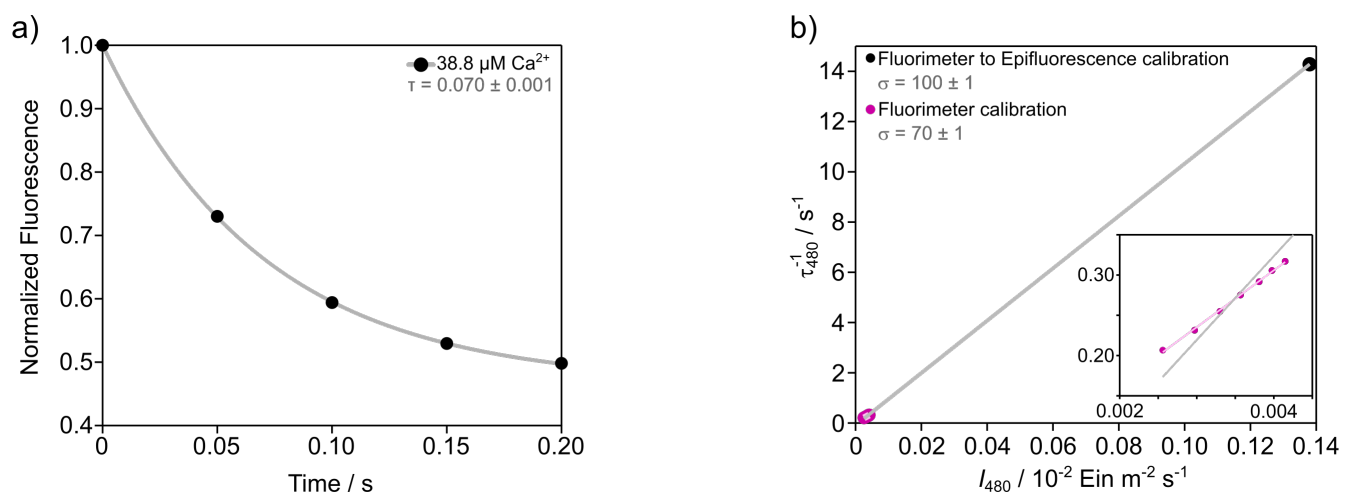

Figure S9: a) Temporal evolution of the normalized fluorescence signal recorded at $515 \mathrm{~nm}$ for GCaMP6s-Q under saturated calcium concentration at $\left[\mathrm{Ca}^{2+}\right]_{\text {free }}=38.8 \mu \mathrm{M}$. Experimental data (black markers) have been fitted monoexponential to retrieve $\tau_{\mathrm{TB} \rightarrow \mathrm{PB}, 480}$ (grey line) . Experiment performed in

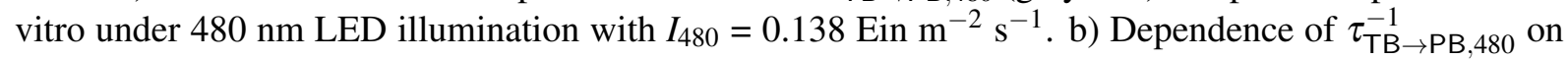
the applied light intensity $I_{480}$ (markers). The photoisomerization cross section $\sigma_{\mathrm{TB} \rightarrow \mathrm{PB}, 480}$ is obtained from the slope of the linear fit. Experiments done in cuvette in the fluorimeter (magenta markers and rose fit) are compared with the one including the experiment under the epifluorescence microscope (black markers and gray fit). A zoom is reported in the inset in the low light intensity regime. [GCaMP6s-Q] = $1 \mu \mathrm{M}$ in $30 \mathrm{mM}$ MOPS, $100 \mathrm{mM} \mathrm{KCl}$ and a buffered concentration of $\left[\mathrm{Ca}^{2+}\right]_{\text {free }}$ (CaEGTA/EGTA), pH $=7.2,20^{\circ} \mathrm{C}$. 


\section{Appendices}

\section{A Theoretical analysis of a reversibly photo-switchable sensor}

\section{A.1 Without illumination}

\section{A.1.1 Thermodynamic aspects}

In the absence of illumination, the reversibly photo-switchable sensor (rs-sensor) is not engaged in any photoisomerization. Its free state $\mathbf{U}$ reacts with its ligand $\mathbf{L}$ to yield a ligand-fully bound state $\mathbf{B}$. We adopt Eq. 9 to account for the thermodynamic aspects of this reaction. $n$ designates the stoichiometric coefficient of the ligand, which defines how many identical ligand molecules bind to the sensor.

$$
n \mathbf{L}+\mathbf{U}=\mathbf{B}
$$

Sensors which bind multiple identical ligands often display positive cooperative binding. Namely, the association constants related to the ligand binding increase after the binding of the first ligand. This effect can be empirically described in the Hill equation (Eq. 10).

$$
\theta=\frac{[\mathbf{L}]^{v}}{\mathrm{Kd}+[\mathbf{L}]^{v}}=\frac{[\mathbf{L}]^{v}}{\left(\mathrm{~K}_{0.5}\right)^{v}+[\mathbf{L}]^{v}}
$$

In Eq. 10, $\theta$ defines the fraction of the sensor that is bound by the ligand, $[\mathbf{L}]$ is the concentration of the free ligand, and $v$ is the Hill coefficient. The apparent dissociation constant, $\mathrm{Kd}$, is related to the microscopic dissociation constant (named also half-saturation constant) as $\mathrm{Kd}^{1 / v}=\mathrm{K}_{0.5} \cdot \mathrm{K}_{0.5}$ defines the ligand concentration, which results in half occupation. Positively cooperative sensor-ligand systems display $v>1$.

\section{A.1.2 Kinetic aspects}

When the system exhibits cooperativity, the mechanism governing the reaction of the sensor with ligands can be complex. In the following, we are concerned with a kinetic model accounting for the time evolution of the sensor signal. We address kinetics at the microscopic level and consider in Eq. 11 a simple kinetic model in which the free sensor, $\mathbf{U}$, yields the ligand-fully bound sensor, $\mathbf{B}$, upon reacting with one ligand molecule.

$$
\mathbf{L}+\mathbf{U} \underset{k_{-\mathbf{L}}(t)}{\stackrel{k_{+\mathbf{L}}(t)}{\rightleftharpoons}} \mathbf{B}
$$

In Eq. 11 , the kinetic constants $k_{-\mathbf{L}}$ and $k_{+\mathbf{L}}$ are associated to the rate-limiting steps determining the time evolution of the sensor signal upon reaction of the ligand with the sensor. In the case later presented on GCaMP6s-Q, the rate-limiting step is presumably the interaction of the RS20 peptide with calmodulin. ${ }^{1,3,5,6}$ 


\section{A.2 Under illumination}

\section{A.2.1 From the eight- to the four-state kinetic model}

When subjected to appropriate illumination, the rs-sensor is submitted to both ligand's association-dissociation reactions and photoisomerizations of the sensor's chromophore. Further considering that rs-sensors based on reversibly photoswitchable fluorescent proteins (RSFPs) commonly experience proton exchanges, we correspondingly adopt a dynamic model involving three categories of processes (see Figure S10): i) isomerizations between the chromophore in its thermodynamically stable $\mathbf{T}$ and photo-activated $\mathbf{P}$ states (where the conversion $\mathbf{T} \rightarrow \mathbf{P}$ is photochemically-driven whereas the conversion $\mathbf{P} \rightarrow \mathbf{T}$ is both photochemically- and thermally-driven); ii) proton exchanges involving the protonated neutral and deprotonated anionic states of the chromophore $\left(\mathbf{T H}\right.$ and $\mathbf{T}^{-}$, and $\mathbf{P H}$ and $\mathbf{P}^{-}$in the $\mathbf{T}$ and $\mathbf{P}$ states respectively), and iii) ligand exchanges at the sensing site between bound and unbound states (THB and THU, and $\mathbf{T}^{-} \mathbf{B}$ and $\mathbf{T}^{-} \mathbf{U}$ on one hand, and $\mathbf{P H B}$ and $\mathbf{P H U}$, and $\mathbf{P}^{-} \mathbf{B}$ and $\mathbf{P}^{-} \mathbf{U}$ on the other hand, when the chromophore is in its $\mathbf{T}$ and $\mathbf{P}$ states respectively).

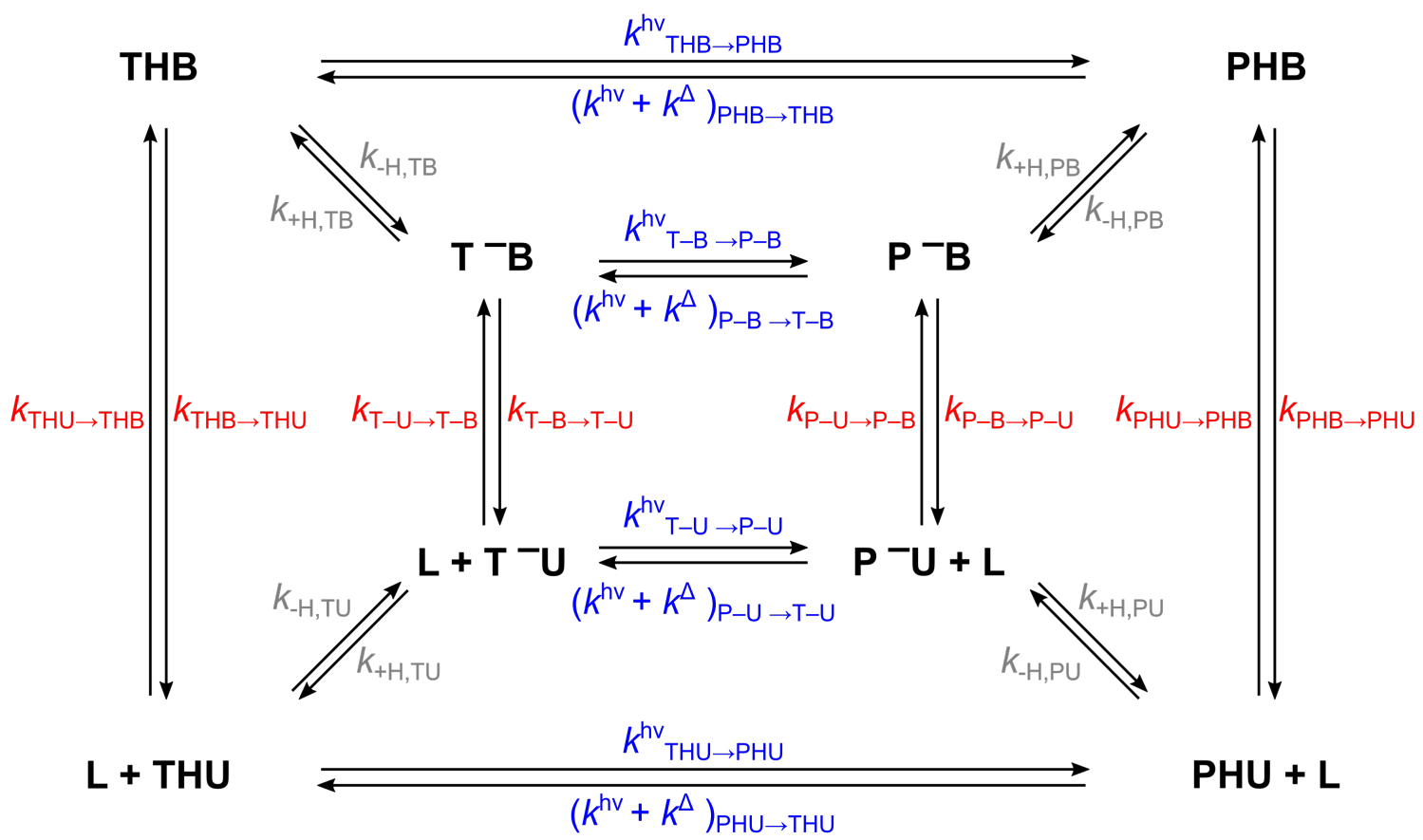

Figure S10: Eight-state kinetic model for a reversibly photo-switchable sensor engaged in proton exchanges at the chromophore with the respective kinetic constants of protonation-deprotonation (grey), ligand association-dissociation (red), and photoisomerization (blue).

The latter eight-state kinetic model does not permit to obtain analytic solutions of the temporal dependence of the concentrations of the reactive states. In order to reach a level at which analytic solutions can be extracted, this detailed model is reduced stepwise by eliminating the fastest steps among the chromophore isomerizations, proton exchanges and complexation reactions.

We take into account that the kinetics of proton exchange are fast (in the range from ns - for standard acids and bases at regular concentrations - up to ms - for the ionizable chromophore of fluorescent proteins $^{4}$ ). At the time scale of our physicochemical experiments, we correspondingly first reduce the eight-state model to a four-state model (Figure S11), in which each state TB, TU, PB, and PU designates 
an average species over the protonated and deprotonated states, which is determined by $\mathrm{pH}$ and each related pKa value. This four-state kinetic model is used in the Main Text for the discussion.

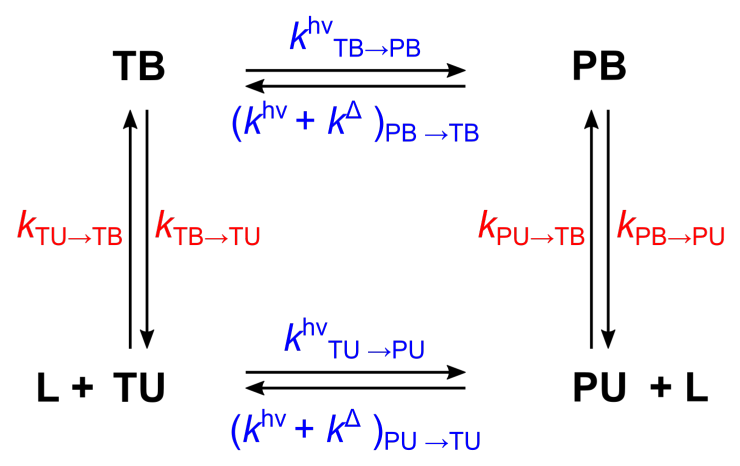

Figure S11: Reduced four-state kinetic model after elimination of the proton exchanges. The respective kinetic constants are depicted for the ligand association-dissociation (red) and photoisomerization (blue) steps.

The concentration profiles in time are governed by the differential equations (12-15):

$$
\begin{aligned}
& \frac{d[\mathbf{T B}]}{d t}=-\left(k_{\mathrm{TB} \rightarrow \mathrm{PB}}^{h v}+k_{\mathrm{TB} \rightarrow \mathrm{TU}}\right)[\mathbf{T B}]+\left(k^{h v}+k^{\Delta}\right)_{\mathrm{PB} \rightarrow \mathrm{TB}}[\mathbf{P B}]+k_{\mathrm{TU} \rightarrow \mathrm{TB}}[\mathbf{T U}][\mathbf{L}] \\
& \frac{d[\mathbf{P B}]}{d t}=-\left\{\left(k^{h v}+k^{\Delta}\right)_{\mathrm{PB} \rightarrow \mathrm{TB}}+k_{\mathrm{PB} \rightarrow \mathrm{PU}}\right\}[\mathbf{P B}]+k_{\mathrm{TB} \rightarrow \mathrm{PB}}^{h v}[\mathbf{T B}]+k_{\mathrm{PU} \rightarrow \mathrm{PB}}[\mathbf{P U}][\mathbf{L}] \\
& \frac{d[\mathbf{T U}]}{d t}=-\left(k_{\mathrm{TU} \rightarrow \mathrm{PU}}^{h v}+k_{\mathrm{TU} \rightarrow \mathrm{TB}}[\mathbf{L}]\right)[\mathbf{T U}]+k_{\mathrm{TB} \rightarrow \mathrm{TU}}[\mathbf{T B}]+\left(k^{h v}+k^{\Delta}\right)_{\mathrm{PU} \rightarrow \mathrm{TU}}[\mathbf{P U}] \\
& \frac{d[\mathbf{P U}]}{d t}=-\left\{\left(k^{h v}+k^{\Delta}\right)_{\mathrm{PU} \rightarrow \mathrm{TU}}+k_{\mathrm{PU} \rightarrow \mathrm{PB}}[\mathbf{L}]\right\}[\mathbf{P U}]+k_{\mathrm{PB} \rightarrow \mathrm{PU}}[\mathbf{P B}]+k_{\mathrm{TU} \rightarrow \mathrm{PU}}^{h v}[\mathbf{T U}]
\end{aligned}
$$

In particular, Eqs. (12-15) can be used to generate the system of equations (16-19), which gives access to the system composition at steady-state upon illumination:

$$
\begin{aligned}
& 0=-\left(k_{\mathrm{TB} \rightarrow \mathrm{PB}}^{h v}+k_{\mathrm{TB} \rightarrow \mathrm{TU}}\right)[\mathbf{T B}]^{\infty}+\left(k^{h v}+k^{\Delta}\right)_{\mathrm{PB} \rightarrow \mathrm{TB}}[\mathbf{P B}]^{\infty}+k_{\mathrm{TU} \rightarrow \mathrm{TB}}[\mathbf{T U}]^{\infty}[\mathbf{L}]^{\infty} \\
& 0=-\left\{\left(k^{h v}+k^{\Delta}\right)_{\mathrm{PB} \rightarrow \mathrm{TB}}+k_{\mathrm{PB} \rightarrow \mathrm{PU}}\right\}[\mathbf{P B}]^{\infty}+k_{\mathrm{TB} \rightarrow \mathrm{PB}}^{h v}[\mathbf{T B}]^{\infty}+k_{\mathrm{PU} \rightarrow \mathrm{PB}}[\mathbf{P U}]^{\infty}[\mathbf{L}]^{\infty} \\
& 0=-\left(k_{\mathrm{TU} \rightarrow \mathrm{PU}}^{h v}+k_{\mathrm{TU} \rightarrow \mathrm{TB}}[\mathbf{L}]^{\infty}\right)[\mathbf{T U}]^{\infty}+\left(k^{h v}+k^{\Delta}\right)_{\mathrm{TB} \rightarrow \mathrm{TU}}[\mathbf{T B}]^{\infty}+k_{\mathrm{PU} \rightarrow \mathrm{TU}}[\mathbf{P U}]^{\infty} \\
& 0=-\left\{\left(k^{h v}+k^{\Delta}\right)_{\mathrm{PU} \rightarrow \mathrm{TU}}+k_{\mathrm{PU} \rightarrow \mathrm{PB}}[\mathbf{L}]^{\infty}\right\}[\mathbf{P U}]^{\infty}+k_{\mathrm{PB} \rightarrow \mathrm{PU}}[\mathbf{P B}]^{\infty}+k_{\mathrm{TU} \rightarrow \mathrm{PU}}^{h v}[\mathbf{T U}]^{\infty}
\end{aligned}
$$

Eqs.(16-19) show that at steady-state, the system is out-of-equilibrium and that its composition is determined by eight rate constants.

\section{A.2.2 From the four- to two-state kinetic models}

Although simpler than the eight-state model, the four-state model still does not allow to analytically solve the temporal dependence of the concentrations of its four species. In contrast, analytic solutions can be found for asymptotic conditions in which the four-state model can be further reduced to two-state exchanges after further averaging over the fastest steps. The two identified asymptotic cases are i) photoisomerizations are slower than ligand complexations and ii) ligand complexations are slower than the photoisomerizations. Crossing between both kinetic regimes typically occurs when the relaxation 
times associated to the photoisomerizations and the complexation reactions are equal. The analysis below is closely similar to what has been reported in the Supporting Information of P. Wang et $a l^{7}$ and we here only provide the key elements.

The photoisomerizations are rate-limiting Under such conditions, the four-state model reduces at the longest time scale to the two-state exchange given in Eq. 20

$$
\mathbf{T} \underset{k_{\mathrm{P} \rightarrow \mathrm{T}}(t)}{\stackrel{k_{\mathrm{T} \rightarrow \mathrm{P}}(t)}{\rightleftharpoons}} \mathbf{P}
$$

involving the average $\mathbf{T}$ over all states in which the chromophore is in its thermodynamically most stable state and the average $\mathbf{P}$ over all states in which the chromophore is in its photo-activated state.

Under illumination involving light intensities $I_{1}(\mathrm{t})$ at wavelength $\lambda_{1}$ and $I_{2}(\mathrm{t})$ at wavelength $\lambda_{2}$, the rate constants $k_{\mathrm{T} \rightarrow \mathrm{P}}(t)$ and $k_{\mathrm{P} \rightarrow \mathrm{T}}(t)$ are given in Eqs. 21,22

$$
\begin{aligned}
& k_{\mathrm{T} \rightarrow \mathrm{P}}(t)=\sigma_{\mathrm{T} \rightarrow \mathrm{P}, 1} I_{1}(t)+\sigma_{\mathrm{T} \rightarrow \mathrm{P}, 2} I_{2}(t) \\
& k_{\mathrm{P} \rightarrow \mathrm{T}}(t)=\sigma_{\mathrm{P} \rightarrow \mathrm{T}, 1} I_{1}(t)+\sigma_{\mathrm{P} \rightarrow \mathrm{T}, 2} I_{2}(t)+k_{\mathrm{P} \rightarrow \mathrm{T}}^{\Delta}
\end{aligned}
$$

where $\sigma_{\mathrm{T} \rightarrow \mathrm{P}, i}$ and $\sigma_{\mathrm{P} \rightarrow \mathrm{T}, i}(i=1,2)$ designate the photoisomerization cross sections respectively associated to the conversions $\mathbf{T} \rightarrow \mathbf{P}$ and $\mathbf{P} \rightarrow \mathbf{T}$ at the wavelength $\lambda_{i}$ and $k_{\mathrm{P} \rightarrow \mathrm{T}}^{\Delta}$ is the thermal contribution to the rate constant $k_{\mathrm{P} \rightarrow \mathrm{T}}(t)$. These five parameters fully define the behavior of the reversibly photo-switchable sensor.

Assuming that the system is homogeneous at any time and uniformly illuminated, the time evolutions of the concentration of the photoisomeric states $[\mathbf{P}]$ and $[\mathbf{T}]$ are defined in Eqs. 23 and 24:

$$
\begin{aligned}
\frac{d[\mathbf{T}]}{d t} & =-k_{\mathrm{T} \rightarrow \mathrm{P}}(t)[\mathbf{T}]+k_{\mathrm{P} \rightarrow \mathrm{T}}(t)[\mathbf{P}] \\
\frac{d[\mathbf{P}]}{d t} & =+k_{\mathrm{T} \rightarrow \mathrm{P}}(t)[\mathbf{T}]-k_{\mathrm{P} \rightarrow \mathrm{T}}(t)[\mathbf{P}]
\end{aligned}
$$

From solving Eqs. 23 and 24, it results that the concentrations $[\mathbf{P}]$ and $[\mathbf{T}]$ exhibit a monoexponential relaxation with $\left(k_{\mathrm{T} \rightarrow \mathrm{P}}+k_{\mathrm{P} \rightarrow \mathrm{T}}\right)^{-1}$ relaxation time towards the steady-state upon constant illumination.

The ligand exchanges are rate-limiting Under such conditions, the four-state model reduces at intermediate time scale to two two-state exchanges given in Eqs. 25 and 26

$$
\begin{gathered}
\text { TB } \frac{k_{\mathrm{TB} \rightarrow \mathrm{PB}}(t)}{\underbrace{}_{k_{\mathrm{PB} \rightarrow \mathrm{TB}}(t)}} \mathbf{P B} \\
\mathbf{T U} \frac{k_{\mathrm{TU} \rightarrow \mathrm{PU}(t)}}{k_{\mathrm{PU} \rightarrow \mathrm{TU}(t)}} \mathbf{P U}
\end{gathered}
$$

and the system further relaxes by ligand exchanges given in Eq. 27 at the longest time scale

$$
\mathbf{B} \underset{k_{U \rightarrow \mathrm{B}}(t)}{\stackrel{k_{\mathrm{B} \rightarrow \mathrm{U}}(t)}{\rightleftharpoons}} \mathbf{U}
$$


where $\mathbf{B}$ and $\mathbf{U}$ designate the averages over all chromophore states in which the sensing site is bound and free, respectively. Assuming again that the system is homogeneous at any time and uniformly illuminated, the time evolutions of the concentration in the sensor states now exhibit a triexponential relaxation $^{2}$ towards the steady-state upon constant illumination.

\section{B Light jump experiments}

In order to acquire kinetic information of GCaMP6s-Q, we performed alternated light jump experiments at two wavelengths: first at $\lambda_{1}$ to drive GCaMP6s- $\mathbf{Q}$ from its $\mathbf{T}$ to its $\mathbf{P}$ state, and second at $\lambda_{2}$ to convert back $\mathbf{P}$ to $\mathbf{T}$. In this section, $\lambda_{1}=480 \mathrm{~nm}$ and $\lambda_{2}=405 \mathrm{~nm}$ and we use below the notations $\lambda_{480}, I_{480}$, $\lambda_{405}$, and $I_{405}$ for clarity.

\section{B.1 Sudden illumination at $\lambda_{480}$}

We address sudden illumination of GCaMP6s-Q to constant light intensity $I(t)=I_{480}$. The forward and backward rate constants of photoisomerization of the sensor become:

$$
\begin{aligned}
& k_{\mathrm{T} \rightarrow \mathrm{P}, 480}=\sigma_{\mathrm{T} \rightarrow \mathrm{P}, 480} I_{480} \\
& k_{\mathrm{P} \rightarrow \mathrm{T}, 480}=\sigma_{\mathrm{P} \rightarrow \mathrm{T}, 480} I_{480}+k_{\mathrm{P} \rightarrow \mathrm{T}}^{\Delta}
\end{aligned}
$$

\section{B.1.1 Rate-limiting photoisomerizations}

We first assume that the photoisomerizations are rate-limiting in the overall relaxation of an illuminated solution of the rs-sensor. Therefore we rely on the two-state model given in Eq. 20 to retrieve the expressions of the time evolution of the state concentrations and fluorescence signal of the sensor.

Time evolution of the concentrations Starting from a solution containing the thermodynamically stable state $\mathbf{T}$ in the absence of any illumination, the concentrations $[\mathbf{T}]$ and $[\mathbf{P}]$ evolve in time as

$$
[\mathbf{P}]-\left[\mathbf{P}_{480}^{\infty}\right]=\left[\mathbf{T}_{480}^{\infty}\right]-[\mathbf{T}]=-\left[\mathbf{P}_{480}^{\infty}\right] \exp \left(-\frac{t}{\tau_{\mathrm{T} \rightarrow \mathrm{P}, 480}}\right)
$$

In Eq. 30, $\left[\mathbf{T}_{480}^{\infty}\right]$ and $\left[\mathbf{P}_{480}^{\infty}\right]$ are the concentrations of $\mathbf{T}$ and $\mathbf{P}$ at the photo-stationary state given in Eq. 31

$$
\left[\mathbf{T}_{480}^{\infty}\right]=\left[\mathbf{S}_{\text {tot }}\right]-\left[\mathbf{P}_{480}^{\infty}\right]=\frac{1}{1+\mathrm{K}_{\mathrm{T} \rightarrow \mathrm{P}}^{480}}\left[\mathbf{S}_{\mathrm{tot}}\right]
$$

with

$$
\mathrm{K}_{\mathrm{T} \rightarrow \mathrm{P}}^{480}=\frac{k_{\mathrm{T} \rightarrow \mathrm{P}, 480}}{k_{\mathrm{P} \rightarrow \mathrm{T}, 480}}=\frac{\sigma_{\mathrm{T} \rightarrow \mathrm{P}, 480} I_{480}}{\sigma_{\mathrm{P} \rightarrow \mathrm{T}, 480} I_{480}+k_{\mathrm{P} \rightarrow \mathrm{T}}^{\Delta}}
$$

\footnotetext{
${ }^{2}$ It can become similar to a biexponential relaxation if two system relaxation times are similar.
} 
and $\left[\mathbf{S}_{\mathrm{tot}}\right]=[\mathbf{T}]+[\mathbf{P}]=\left[\mathbf{T}_{480}^{\infty}\right]+\left[\mathbf{P}_{480}^{\infty}\right]$ the total concentration of the sensor. They are reached after a few times $^{3}$ the relaxation time $\tau_{\mathrm{T} \rightarrow \mathrm{P}, 480}$ defined as

$$
\tau_{\mathrm{T} \rightarrow \mathrm{P}, 480}=\frac{1}{k_{\mathrm{T} \rightarrow \mathrm{P}, 480}+k_{\mathrm{P} \rightarrow \mathrm{T}, 480}}=\frac{1}{\left(\sigma_{\mathrm{T} \rightarrow \mathrm{P}, 480}+\sigma_{\mathrm{P} \rightarrow \mathrm{T}, 480}\right) I_{480}+k_{\mathrm{P} \rightarrow \mathrm{T}}^{\Delta}} .
$$

Time evolution of the fluorescence signal The fluorescence signal $F_{480}(t)$ results from the sum of the individual contributions of the species $\mathbf{T}$ and $\mathbf{P}$. It can be written as

$$
F_{480}(t)=\left[Q_{\mathrm{T}, 480}[\mathbf{T}](t)+Q_{\mathrm{P}, 480}[\mathbf{P}](t)\right] I_{480}(t)
$$

where $Q_{\mathrm{T}, 480}$ and $Q_{\mathrm{P}, 480}$ are the molecular brightnesses at $\lambda_{480}$ of $\mathbf{T}$ and $\mathbf{P}$, respectively.

Eqs. 30 and 34 yield:

$$
\frac{F_{480}(t)}{F_{480}(0)}=1+\frac{\left(Q_{\mathrm{P}, 480}-Q_{\mathrm{T}, 480}\right) I_{480}}{F_{480}(0)} \frac{\mathrm{K}_{\mathrm{T} \rightarrow \mathrm{P}}^{480}}{1+\mathrm{K}_{\mathrm{T} \rightarrow \mathrm{P}}^{480}}\left[\mathbf{S}_{\mathrm{tot}}\right]\left[1-\exp \left(-\frac{t}{\tau_{\mathrm{T} \rightarrow \mathrm{P}, 480}}\right)\right]
$$

where

$$
F_{480}(0)=\left(Q_{\mathrm{T}, 480} I_{480}\right)\left[\mathbf{S}_{\mathrm{tot}}\right]=\left(Q_{\mathrm{T}, 480} I_{480}\right)\left[\mathbf{T}^{0}\right]
$$

and $\left[\mathbf{T}^{0}\right]=\left[\mathbf{S}_{\text {tot }}\right]$ is the initial $\mathbf{T}$ concentration.

Eq. 35 can be used to fit the fluorescence temporal evolution upon $480 \mathrm{~nm}$ illumination. For a given $[\mathbf{L}]$, the fit allows the extraction of the characteristic photoisomerization time $\tau_{\mathrm{T} \rightarrow \mathrm{P}, 480}$. The simplified equation used for fitting is reported in Eq. 2. Thus, the dependence of $\tau_{\top \rightarrow P, 480}$ on $[\mathbf{L}]$ can be used to calibrate the rs-sensor (Hill equation Eq. 1).

Starting from $F_{480}(0)$, the fluorescence emission exponentially decays toward

$$
F_{480}(\infty)=\left[\left(Q_{\mathrm{T}, 480} \frac{1}{1+\mathrm{K}_{\mathrm{T} \rightarrow \mathrm{P}}^{480}}+Q_{\mathrm{P}, 480} \frac{\mathrm{K}_{\mathrm{T} \rightarrow \mathrm{P}}^{480}}{1+\mathrm{K}_{\mathrm{T} \rightarrow \mathrm{P}}^{480}}\right) I_{480}\right]\left[\mathbf{S}_{\mathrm{tot}}\right]
$$

From Eqs. 36 and 37, it yields

$$
F_{480}(0)-F_{480}(\infty)=\left(Q_{\mathrm{T}, 480}-Q_{\mathrm{P}, 480}\right) \frac{\mathrm{K}_{\mathrm{T} \rightarrow \mathrm{P}}^{480}}{1+\mathrm{K}_{\mathrm{T} \rightarrow \mathrm{P}}^{480}} I_{480}\left[\mathbf{S}_{\mathrm{tot}}\right]
$$

The validation of the two-state model given in Eq. 20 is effective from:

- The quality of the fit of the fluorescence signal obtained by using Eq. 35;

- The observation of the linear dependence of $\left(\tau_{\mathrm{T} \rightarrow \mathrm{P}, 480}\right)^{-1}$ on $I_{480}$ predicted in Eq. 33, which enables us to extract $\sigma_{\mathrm{T} \rightarrow \mathrm{P}, 480}+\sigma_{\mathrm{P} \rightarrow \mathrm{T}, 480}$ and $k_{\mathrm{P} \rightarrow \mathrm{T}}^{\Delta}$.

Provided that $\sigma_{\mathrm{P} \rightarrow \mathrm{T}, 480} I_{480} \gg k_{\mathrm{P} \rightarrow \mathrm{T}}^{\Delta}$ (which makes $\mathrm{K}_{\mathrm{T} \rightarrow \mathrm{P}}^{480}$ not to depend on $I_{480}$ ), the observation of the linear dependence of $F_{480}(0)-F_{480}(\infty)$ on $I_{480}$ predicted in Eq. 38 further supports the validation of the

\footnotetext{
${ }^{3} \mathrm{~A}$ time window corresponding to $\mathrm{t}=5 \cdot \tau_{\mathrm{T} \rightarrow \mathrm{P}, 480}$ ensures full relaxation to the photo-steady state.
} 
two-state model given in Eq. 20. Conversely, if $\sigma_{\mathrm{P} \rightarrow \mathrm{T}, 480} I_{480} \leq k_{\mathrm{P} \rightarrow \mathrm{T}}^{\Delta}$ (which makes $\mathrm{K}_{\mathrm{T} \rightarrow \mathrm{P}}^{480}$ to depend on $\left.I_{480}\right)$, the linear dependence of $F_{480}(0)-F_{480}(\infty)$ on $I_{480}$, indicates that $\mathrm{K}_{\mathrm{T} \rightarrow \mathrm{P}}^{480} \gg 1$ and Eq. 38 can be then simplified to:

$$
F_{480}(0)-F_{480}(\infty)=\left(Q_{\mathrm{T}, 480}-Q_{\mathrm{P}, 480}\right) I_{480}\left[\mathbf{S}_{\mathrm{tot}}\right]
$$

Therefore, based on Eqs. 36 and 39, the empirical value $\Delta F / F(0)$ previously used to calibrate the rs-sensor ${ }^{2}$ evaluates the brightness variation upon analyte titration and does not contain any kinetic information (Eq. 40).

$$
\frac{\Delta F}{F(0)}=\frac{F_{480}(0)-F_{480}(\infty)}{F(0)}=1-\frac{Q_{\mathrm{P}, 480}}{Q_{\mathrm{T}, 480}}
$$

Asymptotic molecular brightness The sensor brightness in its thermodynamically stable state, $Q_{\mathrm{T}, 480}$, can be measured via conventional methods based on the molar absorption coefficient, $\varepsilon$, and the fluorescence quantum yield, $\Psi$, based on Eq. 41:

$$
Q_{\mathrm{T}, 480}=\varepsilon_{\mathrm{T}, 480} \Psi_{\mathrm{T}, 480}
$$

In contrast, the sensor brightness in its photo-activated state, $Q_{\mathrm{P} .480}$, cannot be retrieved from only analyzing the time evolution of the fluorescence signal under illumination when the constant $\mathrm{K}_{\mathrm{T} \rightarrow \mathrm{P}}^{480}$ cannot be independently evaluated. Indeed, when the two-state model is relevant, Eqs. 32,33,35 show that the exponential fit provides only two equations linking three unknown quantities $-Q_{\mathrm{P}, 480}, \mathrm{~K}_{\mathrm{T} \rightarrow \mathrm{P}}^{480}$, and $\sigma_{\mathrm{P} \rightarrow \mathrm{T}, 480}$ - with the experimentally accessible relaxation time $\tau_{\mathrm{T} \rightarrow \mathrm{P}, 480}$ and amplitude $F_{480}(0)-F_{480}(\infty)$ of the exponential decay. Yet, once the relevance of the two-state model is established, it is possible to extract a range of $Q_{\mathrm{P}, 480}$ values from exploiting the experimentally accessible parameters $\tau_{\mathrm{T} \rightarrow \mathrm{P}, 480}$ and amplitude $F_{480}(0)-F_{480}(\infty)$ under two asymptotic situations, which arise from exploiting Eq. 42 obtained after combining Eq. 36 and Eq. 37

$$
Q_{\mathrm{P}, 480}=Q_{\mathrm{T}, 480}\left(\frac{1}{R}-\frac{1}{1+\mathrm{K}_{\mathrm{T} \rightarrow \mathrm{P}}^{480}}\right) \frac{1+\mathrm{K}_{\mathrm{T} \rightarrow \mathrm{P}}^{480}}{\mathrm{~K}_{\mathrm{T} \rightarrow \mathrm{P}}^{480}}
$$

where $R=\frac{F_{480}(0)}{F_{480}(\infty)}$. Eq. 42 shows that $Q_{\mathrm{P}, 480}$ monotonously grows with $\mathrm{K}_{\mathrm{T} \rightarrow \mathrm{P}}^{480}$. Therefore the range of $Q_{\mathrm{P}, 480}$ can be retrieved from the accessible range of $\mathrm{K}_{\mathrm{T} \rightarrow \mathrm{P}}^{480}$.

- The lower value of $\mathrm{K}_{\mathrm{T} \rightarrow \mathrm{P}}^{480}$, $\operatorname{Min}\left(\mathrm{K}_{\mathrm{T} \rightarrow \mathrm{P}}^{480}\right)=R-1$, can be extracted from Eq. 42: since $Q_{\mathrm{P}, 480}$ is positive, $\mathrm{K}_{\mathrm{T} \rightarrow \mathrm{P}}^{480} \geq R-1$. For $\mathrm{K}_{\mathrm{T} \rightarrow \mathrm{P}}^{480}=\operatorname{Min}\left(\mathrm{K}_{\mathrm{T} \rightarrow \mathrm{P}}^{480}\right), Q_{\mathrm{P}, 480}=\operatorname{Min}\left(Q_{\mathrm{P}, 480}\right)=0$;

- The upper value of $\mathrm{K}_{\mathrm{T} \rightarrow \mathrm{P}}^{480}, \operatorname{Max}\left(\mathrm{K}_{\mathrm{T} \rightarrow \mathrm{P}}^{480}\right)=\sigma_{\mathrm{T} \rightarrow \mathrm{P}, 480} I_{480} / k_{\mathrm{P} \rightarrow \mathrm{T}}^{\Delta}$, can be extracted from Eq. 32: since $\sigma_{\mathrm{P} \rightarrow \mathrm{T}, 480}$ is positive, $\mathrm{K}_{\mathrm{T} \rightarrow \mathrm{P}}^{480} \leq \sigma_{\mathrm{T} \rightarrow \mathrm{P}, 480} I_{480} / k_{\mathrm{P} \rightarrow \mathrm{T}}^{\Delta}$. For $\mathrm{K}_{\mathrm{T} \rightarrow \mathrm{P}}^{480}=\operatorname{Max}\left(\mathrm{K}_{\mathrm{T} \rightarrow \mathrm{P}}^{480}\right)$, $Q_{\mathrm{P}, 480}=\operatorname{Max}\left(Q_{\mathrm{P}, 480}\right)$, which is obtained by introducing $\operatorname{Max}\left(\mathrm{K}_{\mathrm{T} \rightarrow \mathrm{P}}^{480}\right)$ in Eq. 42.

Therefore, Eqs. 32 and 42 yield boundary values of the photoisomerization constant and molecular brightness for a system that can be reliably described by a two-state model. However it is important to notice that the latter values depend on the light intensity, which figures as parameter both in Eq. 32 - for the calculation of $\mathrm{K}_{\mathrm{T} \rightarrow \mathrm{P}}^{480}$ - and in Eq. 42 , in the expression of $R$ - for the extraction of $Q_{\mathrm{P}, 480}$. 
Nevertheless, for systems that can be described by a two-state model and additionally display linear dependence of $F_{480}(0)-F_{480}(\infty)$ on $I_{480}, Q_{\mathrm{P}, 480}$ can be retrieved from exploiting the slope of the linear dependence of Eq. 38 (for $\sigma_{\mathrm{P} \rightarrow \mathrm{T}, 480} I_{480} \gg k_{\mathrm{P} \rightarrow \mathrm{T}}^{\Delta}$ ) or Eq. 39 (for $\mathrm{K}_{\mathrm{T} \rightarrow \mathrm{P}}^{480} \gg 1$ ) on $I_{480}$. Then $Q_{\mathrm{P}, 480}$ so obtained is independent of $R$ and calibrated over the whole regime of light intensity investigated.

\section{B.1.2 Rate-limiting ligand exchanges}

In this situation, under illumination and at intermediate time scale, the ligand-unbound, $\mathbf{U}$, and -bound, $\mathbf{B}$, states of the rs-sensor evolve independently between their thermodynamically stable (either TU or $\mathbf{T B}$ ) and photo-activated (either PU or PB) states. We rely on the two-state model given in Eq. 20 to retrieve the expressions of the time evolution of the concentrations and fluorescence signal of the ligand-free and -bound states of the sensor.

Time evolution of the concentrations Starting from a solution containing the thermodynamically stable states TX $\left(\mathrm{X}=\mathrm{B}\right.$ or $\mathrm{U}$; at respective concentrations $[\mathbf{T B}]=\theta\left[\mathbf{S}_{\text {tot }}\right]$ and $\left.[\mathbf{T U}]=(1-\theta)\left[\mathbf{S}_{\text {tot }}\right]\right)$ in the absence of any illumination, the concentrations $[\mathbf{T X}]$ and $[\mathbf{P X}]$ evolve in time as

$$
[\mathbf{P X}]-\left[\mathbf{P X}_{480}^{\text {end }}\right]=\left[\mathbf{T X}_{480}^{\text {end }}\right]-[\mathbf{T X}]=-\left[\mathbf{P X} \mathbf{X}_{480}^{\text {end }}\right] \exp \left(-\frac{t}{\tau_{\mathrm{TX} \rightarrow \mathrm{PX}, 480}}\right)
$$

In Eq. 43, $\left[\mathbf{T X}_{480}^{\text {end }}\right]$ and $\left[\mathbf{P X}_{480}^{\text {end }}\right]$ are the concentrations of $\mathbf{T X}$ and $\mathbf{P X}$ at the end of the regime of intermediate times given in Eqs. 44 and $45^{4}$

$$
\begin{gathered}
{\left[\mathbf{T B}_{480}^{\text {end }}\right]=\theta\left[\mathbf{S}_{\mathrm{tot}}\right]-\left[\mathbf{P B}_{480}^{\infty}\right]=\frac{1}{1+\mathrm{K}_{\mathrm{TB} \rightarrow \mathrm{PB}}^{480}} \theta\left[\mathbf{S}_{\mathrm{tot}}\right]} \\
{\left[\mathbf{T U}_{480}^{\text {end }}\right]=(1-\theta)\left[\mathbf{S}_{\mathrm{tot}}\right]-\left[\mathbf{P U}_{480}^{\infty}\right]=\frac{1}{1+\mathrm{K}_{\mathrm{TU} \rightarrow \mathrm{PU}}^{480}}(1-\theta)\left[\mathbf{S}_{\mathrm{tot}}\right]}
\end{gathered}
$$

by introducing

$$
\begin{aligned}
& \mathrm{K}_{\mathrm{TB} \rightarrow \mathrm{PB}}^{480}=\frac{k_{\mathrm{TB} \rightarrow \mathrm{PB}, 480}}{k_{\mathrm{PB} \rightarrow \mathrm{TB}, 480}}=\frac{\sigma_{\mathrm{TB} \rightarrow \mathrm{PB}, 480} I_{480}}{\sigma_{\mathrm{PB} \rightarrow \mathrm{TB}, 480} I_{480}+k_{\mathrm{PB} \rightarrow \mathrm{TB}}^{\Delta}} \\
& \mathrm{K}_{\mathrm{TU} \rightarrow \mathrm{PU}}^{480}=\frac{k_{\mathrm{TU} \rightarrow \mathrm{PU}, 480}}{k_{\mathrm{PU} \rightarrow \mathrm{TU}, 480}}=\frac{\sigma_{\mathrm{TU} \rightarrow \mathrm{PU}, 480} I_{480}}{\sigma_{\mathrm{PU} \rightarrow \mathrm{TU}, 480} I_{480}+k_{\mathrm{PU} \rightarrow \mathrm{TU}}^{\Delta}}
\end{aligned}
$$

which are respectively reached after a few times the relaxation times $\tau_{\mathrm{TB} \rightarrow \mathrm{PB}, 480}$ and $\tau_{\mathrm{TU} \rightarrow \mathrm{PU}, 480}$ defined as

$$
\tau_{\mathrm{TB} \rightarrow \mathrm{PB}, 480}=\frac{1}{k_{\mathrm{TB} \rightarrow \mathrm{PB}, 480}+k_{\mathrm{TB} \rightarrow \mathrm{PB}, 480}}=\frac{1}{\left(\sigma_{\mathrm{TB} \rightarrow \mathrm{PB}, 480}+\sigma_{\mathrm{PB} \rightarrow \mathrm{TB}, 480}\right) I_{480}+k_{\mathrm{PB} \rightarrow \mathrm{TB}}^{\Delta}}
$$

\footnotetext{
${ }^{4}$ To emphasize that data processing involves a kinetic regime of intermediate times, we adopted the notation end instead of $\infty$ to indicate the final value observed at the end of the investigated time window.
} 


$$
\tau_{\mathrm{TU} \rightarrow \mathrm{PU}, 480}=\frac{1}{k_{\mathrm{TU} \rightarrow \mathrm{PU}, 480}+k_{\mathrm{TU} \rightarrow \mathrm{PU}, 480}}=\frac{1}{\left(\sigma_{\mathrm{TU} \rightarrow \mathrm{PU}, 480}+\sigma_{\mathrm{PU} \rightarrow \mathrm{TU}, 480}\right) I_{480}+k_{\mathrm{PU} \rightarrow \mathrm{TU}}^{\Delta}}
$$

Time evolution of the fluorescence signal The fluorescence signal $F_{480}(t)$ results from the sum of the individual contributions of the species $\mathbf{T}$ and $\mathbf{P}$. It can be written as

$$
F_{480}(t)=\left\{\left[Q_{\mathrm{TB}, 480}[\mathbf{T B}](t)+Q_{\mathrm{PB}, 480}[\mathbf{P B}](t)\right]+\left[Q_{\mathrm{TU}, 480}[\mathbf{T U}](t)+Q_{\mathrm{PU}, 480}[\mathbf{P U}](t)\right]\right\} I_{480}(t)
$$

where $Q_{\mathrm{TX}, 480}$ and $Q_{\mathrm{PX}, 480}$ are the molecular brightnesses at $\lambda_{480}$ of $\mathbf{T X}$ and $\mathbf{P X}$, respectively.

Eqs. 43 and 50 yield:

$$
\begin{array}{r}
\frac{F_{480}(t)}{F_{480}(0)}=1+\frac{\left(Q_{\mathrm{PB}, 480}-Q_{\mathrm{TB}, 480}\right) I_{480}}{F(0)} \frac{\mathrm{K}_{\mathrm{TB} \rightarrow \mathrm{PB}}^{480}}{1+\mathrm{K}_{\mathrm{TB} \rightarrow \mathrm{PB}}^{480}} \theta\left[\mathbf{S}_{\mathrm{tot}}\right]\left[1-\exp \left(-\frac{t}{\tau_{\mathrm{TB} \rightarrow \mathrm{PB}, 480}}\right)\right] \\
+\frac{\left(Q_{\mathrm{PU}, 480}-Q_{\mathrm{TU}, 480}\right) I_{480}}{F(0)} \frac{\mathrm{K}_{\mathrm{TU} \rightarrow \mathrm{PU}}^{480}}{1+\mathrm{K}_{\mathrm{TU} \rightarrow \mathrm{PU}}^{480}}(1-\theta)\left[\mathbf{S}_{\mathrm{tot}}\right]\left[1-\exp \left(-\frac{t}{\tau_{\mathrm{TU} \rightarrow \mathrm{PU}, 480}}\right)\right]
\end{array}
$$

where

$$
F_{480}(0)=\left[Q_{\mathrm{TB}, 480} \theta+Q_{\mathrm{TU}, 480}(1-\theta)\right]\left[\mathbf{S}_{\mathrm{tot}}\right] I_{480}
$$

Starting form $F_{480}(0)$, the fluorescence emission exponentially decays toward

$F_{480}($ end $)=\left[\left(\frac{Q_{\mathrm{TB}, 480}}{1+\mathrm{K}_{\mathrm{TB} \rightarrow \mathrm{PB}}^{480}}+\frac{\mathrm{K}_{\mathrm{TB} \rightarrow \mathrm{PB}}^{480} Q_{\mathrm{PB}, 480}}{1+\mathrm{K}_{\mathrm{TB} \rightarrow \mathrm{PB}}^{480}}\right) \boldsymbol{\theta}+\left(\frac{Q_{\mathrm{TU}, 480}}{1+\mathrm{K}_{\mathrm{TU} \rightarrow \mathrm{PU}}^{480}}+\frac{\mathrm{K}_{\mathrm{TU} \rightarrow \mathrm{PU}}^{480} Q_{\mathrm{PU}, 480}}{1+\mathrm{K}_{\mathrm{TU} \rightarrow \mathrm{PU}}^{480}}\right)(1-\boldsymbol{\theta})\right]\left[\mathbf{S}_{\mathrm{tot}} I_{480}\right.$

Eq. 51 can be used to fit the fluorescence temporal evolution upon $480 \mathrm{~nm}$ illumination as a function of the analyte concentration. For a given $[\mathbf{L}]$, the fit allows the extraction of the bound fraction $(\theta)$ based on the known characteristic times and the amplitudes of photoisomerization at asymptotic $[\mathbf{L}]$. The simplified equation used for fitting is reported in Eqs. 4 and 6. Thus, the dependence of $\theta$ on $[\mathbf{L}]$ can be fitted with Hill equation Eq. 1, 10.

From Eqs. 52 and 53, it yields

$F_{480}(0)-F_{480}($ end $)=\left[\left(Q_{\mathrm{TB}, 480}-Q_{\mathrm{PB}, 480}\right) \frac{\mathrm{K}_{\mathrm{T} \rightarrow \mathrm{P}}^{480}}{1+\mathrm{K}_{\mathrm{TB} \rightarrow \mathrm{PB}}^{80}} \theta+\left(Q_{\mathrm{TU}, 480}-Q_{\mathrm{PU}, 480}\right) \frac{\mathrm{K}_{\mathrm{TU} \rightarrow \mathrm{PU}}^{480}}{1+\mathrm{K}_{\mathrm{TU} \rightarrow \mathrm{PU}}^{480}}(1-\theta)\right] I_{480}\left[\mathbf{S}_{\mathrm{tot}}\right]$

Interestingly, when $Q_{\mathrm{PU}, 480} \simeq Q_{\mathrm{TU}, 480}$ as in our experimental system, Eq. 51 becomes

$$
\frac{F_{480}(t)}{F_{480}(0)}=1+\frac{\left(Q_{\mathrm{PB}, 480}-Q_{\mathrm{TB}, 480}\right) I_{480}}{F(0)} \frac{\mathrm{K}_{\mathrm{TB} \rightarrow \mathrm{PB}}^{480}}{1+\mathrm{K}_{\mathrm{TB} \rightarrow \mathrm{PB}}^{480}} \theta\left[\mathbf{S}_{\mathrm{tot}}\right]\left[1-\exp \left(-\frac{t}{\tau_{\mathrm{TB} \rightarrow \mathrm{PB}, 480}}\right)\right]
$$

and

$$
F_{480}(0)-F_{480}(\text { end })=\left(Q_{\mathrm{TB}, 480}-Q_{\mathrm{PB}, 480}\right) \frac{\mathrm{K}_{\mathrm{T} \rightarrow \mathrm{P}}^{480}}{1+\mathrm{K}_{\mathrm{TB} \rightarrow \mathrm{PB}}^{480}} \theta I_{480}\left[\mathbf{S}_{\mathrm{tot}}\right]
$$


Under this condition, Eq. 55 can be used to fit the fluorescence temporal evolution upon $480 \mathrm{~nm}$ illumination as a function of the analyte concentration. For a given $[\mathbf{L}]$, the fit allows the extraction of the bound fraction $(\theta)$ based on the characteristic times and the amplitudes of photoisomerization at saturated conditions. The simplified equation used for fitting is reported in Eqs. 5 and 7. Thus, the dependence of $\theta$ on $[\mathbf{L}]$ can be used to calibrate the rs-sensor (Hill equation Eq. 1).

Asymptotic molecular brightness The sensor brightness in its thermodynamically stable ligand-bound and -free states, $Q_{\mathrm{TB}, 480}$ and $Q_{\mathrm{TU}, 480}$, can be extracted during the titration with the ligand via conventional methods based on the molar absorption coefficient, $\varepsilon$, and the fluorescence quantum yield, $\Psi$, based on the following equations:

$$
\begin{aligned}
Q_{\mathrm{TB}, 480} & =\varepsilon_{\mathrm{TB}, 480} \Psi_{\mathrm{TB}, 480} \\
Q_{\mathrm{TU}, 480} & =\varepsilon_{\mathrm{TU}, 480} \Psi_{\mathrm{TU}, 480}
\end{aligned}
$$

Moreover, the ratio $\frac{\Delta F_{480}}{F_{480}(0)}=\frac{F_{480}(0)-F_{480}(\text { end })}{F_{480}(0)}$ is

$$
\frac{\Delta F_{480}}{F_{480}(0)}=\frac{\left[\left(Q_{\mathrm{TB}, 480}-Q_{\mathrm{PB}, 480}\right) \frac{\mathrm{K}_{\mathrm{TB}}^{480} \mathrm{~PB}}{1+\mathrm{K}_{\mathrm{TB} \rightarrow \mathrm{PB}}^{480}} \theta+\left(Q_{\mathrm{TU}, 480}-Q_{\mathrm{PU}, 480}\right) \frac{\mathrm{K}_{\mathrm{T}}^{480} \rightarrow \mathrm{PU}}{1+\mathrm{K}_{\mathrm{TU} \rightarrow \mathrm{PU}}^{480}}(1-\theta)\right]}{\left[Q_{\mathrm{TB}, 480} \theta+Q_{\mathrm{TU}, 480}(1-\theta)\right]},
$$

which does not depend on light intensity $I_{480}$ when $\sigma_{\mathrm{P} \rightarrow \mathrm{T}, 480} I_{480} \gg k_{\mathrm{P} \rightarrow \mathrm{T}}^{\Delta}$ or when $\mathrm{K}_{\mathrm{TB} \rightarrow \mathrm{PB}}^{480}$ and $\mathrm{K}_{\mathrm{TU} \rightarrow \mathrm{PU}}^{480} \gg 1$.

\section{B.2 Sudden change of illumination from $\lambda_{480}$ to $\lambda_{405}$}

We first assume that the fluorescence signal does not evolve anymore following the applied illumination with $I_{480}$ as described in the section above. Then it is suddenly submitted to a change of illumination from $I=I_{480}$ to $I=I_{405}$ by turning off light at $\lambda_{480}$ and turning on light at $\lambda_{405}$. During this second kinetic regime, the forward and backward rate constants are:

$$
\begin{aligned}
k_{\mathrm{T} \rightarrow \mathrm{P}, 405} & =\sigma_{\mathrm{T} \rightarrow \mathrm{P}, 405} I_{405} \\
k_{\mathrm{P} \rightarrow \mathrm{T}, 405} & =\sigma_{\mathrm{P} \rightarrow \mathrm{T}, 405} I_{405}+k_{\mathrm{P} \rightarrow \mathrm{T}}^{\Delta}
\end{aligned}
$$

\section{B.2.1 Rate-limiting photoisomerizations}

We first assume that the photoisomerizations are rate-limiting in the overall relaxation of an illuminated solution of the reversibly photo-switchable sensor. Therefore we rely on the two-state model given in Eq. 20 to retrieve the expressions of the time evolution of the concentrations and fluorescence signal of the sensor. 
Time evolution of the concentrations Starting from a solution at steady-state under illumination with $I_{480}$, the concentrations $[\mathbf{T}]$ and $[\mathbf{P}]$ evolve in time as:

$$
[\mathbf{P}]-\left[\mathbf{P}_{405}^{\infty}\right]=\left[\mathbf{T}_{405}^{\infty}\right]-[\mathbf{T}]=\left(\left[\mathbf{P}_{480}^{\infty}\right]-\left[\mathbf{P}_{405}^{\infty}\right]\right) \exp \left(-\frac{t}{\tau_{\mathrm{T} \rightarrow \mathrm{P}, 405}}\right)
$$

where

$$
\tau_{\mathrm{T} \rightarrow \mathrm{P}, 405}=\frac{1}{k_{\mathrm{T} \rightarrow \mathrm{P}, 405}+k_{\mathrm{P} \rightarrow \mathrm{T}, 405}}=\frac{1}{\left(\sigma_{\mathrm{T} \rightarrow \mathrm{P}, 405}+\sigma_{\mathrm{P} \rightarrow \mathrm{T}, 405}\right) I_{405}+k_{\mathrm{P} \rightarrow \mathrm{T}}^{\Delta}}
$$

defines the relaxation time of the reversibly photo-switchable sensor in the second illumination regime and $\left[\mathbf{T}_{405}^{\infty}\right]$ and $\left[\mathbf{P}_{405}^{\infty}\right]$ are the concentrations of $\mathbf{T}$ and $\mathbf{P}$ at the second photo-stationary state under illumination at $405 \mathrm{~nm}$ typically reached after a few $\tau_{\mathrm{T} \rightarrow \mathrm{P}, 405}$

$$
\left[\mathbf{T}_{405}^{\infty}\right]=\left[\mathbf{S}_{\text {tot }}\right]-\left[\mathbf{P}_{405}^{\infty}\right]=\frac{1}{1+\mathrm{K}_{\mathrm{T} \rightarrow \mathrm{P}}^{405}}\left[\mathbf{S}_{\text {tot }}\right]
$$

with

$$
\mathrm{K}_{\mathrm{T} \rightarrow \mathrm{P}}^{405}=\frac{k_{\mathrm{T} \rightarrow \mathrm{P}, 405}}{k_{\mathrm{P} \rightarrow \mathrm{T}, 405}}
$$

Time evolution of the fluorescence signal Eqs. 34, 64, and 62 yield

$$
\frac{F_{405}(t)}{F_{405}(0)}=1+\frac{\left(Q_{\mathrm{P}, 405}-Q_{\mathrm{T}, 405}\right) I_{405}}{F_{405}(0)}\left(\frac{\mathrm{K}_{\mathrm{T} \rightarrow \mathrm{P}}^{405}}{1+\mathrm{K}_{\mathrm{T} \rightarrow \mathrm{P}}^{405}}-\frac{\mathrm{K}_{\mathrm{T} \rightarrow \mathrm{P}}^{480}}{1+\mathrm{K}_{\mathrm{T} \rightarrow \mathrm{P}}^{480}}\right)\left[\mathbf{S}_{\mathrm{tot}}\right]\left[1-\exp \left(-\frac{t}{\tau_{\mathrm{T} \rightarrow \mathrm{P}, 405}}\right)\right]
$$

where

$$
F_{405}(0)=\left(Q_{\mathrm{T}, 405}\left[\mathbf{T}_{480}^{\infty}\right]+Q_{\mathrm{P}, 405}\left[\mathbf{P}_{480}^{\infty}\right]\right) I_{405}
$$

Starting from $F_{405}(0)$, the fluorescence emission exponentially decays toward

$$
F_{405}(\infty)=\left(Q_{\mathrm{T}, 405}\left[\mathbf{T}_{405}^{\infty}\right]+Q_{\mathrm{P}, 405}\left[\mathbf{P}_{405}^{\infty}\right]\right) I_{405}
$$

Eq. 66 can be used to fit the fluorescence temporal evolution upon $405 \mathrm{~nm}$ illumination as a function of the analyte concentration. For a given $[\mathbf{L}]$, the fit allows the extraction of the characteristic photoisomerization time $\tau_{\mathrm{T} \rightarrow \mathrm{P}, 405}$. The simplified equation used for fitting is reported in Eq. 2. Thus, the dependence of $\tau_{\mathrm{T} \rightarrow \mathrm{P}, 480}$ on $[\mathbf{L}]$ can be used to calibrate the rs-sensor (Hill equation Eq. 1).

\section{B.2.2 Rate-limiting ligand exchanges}

In this situation, the ligand-free and -bound states of the reversibly photo-switchable sensor evolve independently under illumination at intermediate time scale. We rely on the two-state model given in Eq. 20 to retrieve the expressions of the time evolution of the concentrations and fluorescence signal of the ligand-free and -bound states of the sensor. 
Time evolution of the concentrations Starting from a solution whose fluorescence signal does not evolve anymore under illumination at $480 \mathrm{~nm}$, the concentrations [TX] and [PX] evolve in time as

$$
[\mathbf{P X}]-\left[\mathbf{P X}_{405}^{\text {end }}\right]=\left[\mathbf{T X}_{405}^{\text {end }}\right]-[\mathbf{T X}]=\left(\left[\mathbf{P X} \mathbf{X}_{480}^{\text {end }}\right]-\left[\mathbf{P X}_{405}^{\text {end }}\right]\right) \exp \left(-\frac{t}{\tau_{\mathrm{TX} \rightarrow \mathrm{PX}, 405}}\right)
$$

In Eq. 69, $\left[\mathbf{T X}_{405}^{\text {end }}\right]$ and $\left[\mathbf{P X}_{405}^{\text {end }}\right]$ are the concentrations of $\mathbf{T X}$ and $\mathbf{P X}$ at the end of the regime of intermediate times given in Eqs. 70 and 71

$$
\begin{gathered}
{\left[\mathbf{T B}_{405}^{\text {end }}\right]=\theta_{480}\left[\mathbf{S}_{\mathrm{tot}}\right]-\left[\mathbf{P B}_{405}^{\text {end }}\right]=\frac{1}{1+\mathrm{K}_{\mathrm{TB} \rightarrow \mathrm{PB}}^{405}} \theta_{480}\left[\mathbf{S}_{\mathrm{tot}}\right]} \\
{\left[\mathbf{T U}_{405}^{\text {end }}\right]=\left(1-\theta_{480}\right)\left[\mathbf{S}_{\mathrm{tot}}\right]-\left[\mathbf{P U}_{405}^{\text {end }}\right]=\frac{1}{1+\mathrm{K}_{\mathrm{TU} \rightarrow \mathrm{PU}}^{405}\left(1-\theta_{480}\right)\left[\mathbf{S}_{\mathrm{tot}}\right]}}
\end{gathered}
$$

by introducing $\theta_{480}$ to parameter the occupation of the binding site of the sensor at steady-state under illumination at $480 \mathrm{~nm}$ and

$$
\begin{aligned}
& \mathrm{K}_{\mathrm{TB} \rightarrow \mathrm{PB}}^{405}=\frac{k_{\mathrm{TB} \rightarrow \mathrm{PB}, 405}}{k_{\mathrm{PB} \rightarrow \mathrm{TB}, 405}}=\frac{\sigma_{\mathrm{TB} \rightarrow \mathrm{PB}, 405} I_{405}}{\sigma_{\mathrm{PB} \rightarrow \mathrm{TB}, 405} I_{405}+k_{\mathrm{PB} \rightarrow \mathrm{TB}}^{\Delta}} \\
& \mathrm{K}_{\mathrm{TU} \rightarrow \mathrm{PU}}^{405}=\frac{k_{\mathrm{TU} \rightarrow \mathrm{PU}, 405}}{k_{\mathrm{PU} \rightarrow \mathrm{TU}, 405}}=\frac{\sigma_{\mathrm{TU} \rightarrow \mathrm{PU}, 405} I_{405}}{\sigma_{\mathrm{PU} \rightarrow \mathrm{TU}, 405} I_{405}+k_{\mathrm{PU} \rightarrow \mathrm{TU}}^{\Delta}}
\end{aligned}
$$

which are respectively reached after a few times the relaxation times $\tau_{\mathrm{TB} \rightarrow \mathrm{PB}, 405}$ and $\tau_{\mathrm{TU} \rightarrow \mathrm{PU}, 405}$ defined as

$$
\begin{gathered}
\tau_{\mathrm{TB} \rightarrow \mathrm{PB}, 405}=\frac{1}{k_{\mathrm{TB} \rightarrow \mathrm{PB}, 405}+k_{\mathrm{TB} \rightarrow \mathrm{PB}, 405}}=\frac{1}{\left(\sigma_{\mathrm{TB} \rightarrow \mathrm{PB}, 405}+\sigma_{\mathrm{PB} \rightarrow \mathrm{TB}, 405}\right) I_{405}+k_{\mathrm{PB} \rightarrow \mathrm{TB}}^{\Delta}} \\
\tau_{\mathrm{TU} \rightarrow \mathrm{PU}, 405}=\frac{1}{k_{\mathrm{TU} \rightarrow \mathrm{PU}, 405}+k_{\mathrm{TU} \rightarrow \mathrm{PU}, 405}}=\frac{1}{\left(\sigma_{\mathrm{TU} \rightarrow \mathrm{PU}, 405}+\sigma_{\mathrm{PU} \rightarrow \mathrm{TU}, 405}\right) I_{405}+k_{\mathrm{PU} \rightarrow \mathrm{TU}}^{\Delta}} .
\end{gathered}
$$

Time evolution of the fluorescence signal The fluorescence signal $F_{405}(t)$ results from the sum of the individual contributions of the species $\mathbf{T}$ and $\mathbf{P}$. It can be written as

$$
F_{405}(t)=\left\{\left[Q_{\mathrm{TB}, 405}[\mathbf{T B}](t)+Q_{\mathrm{PB}, 405}[\mathbf{P B}](t)\right]+\left[Q_{\mathrm{TU}, 405}[\mathbf{T U}](t)+Q_{\mathrm{PU}, 405}[\mathbf{P U}](t)\right]\right\} I_{405}(t)
$$

where $Q_{\mathrm{TX}, 405}$ and $Q_{\mathrm{PX}, 405}$ are the molecular brightnesses at $\lambda_{405}$ of $\mathbf{T X}$ and $\mathbf{P X}$, respectively.

Eqs. 69 and 76 yield:

$$
\begin{array}{r}
\frac{F_{405}(t)}{F_{405}(0)}=1+\frac{\left(Q_{\mathrm{PB}, 405}-Q_{\mathrm{TB}, 405}\right) I_{405}}{F_{405}(0)}\left(\frac{\mathrm{K}_{\mathrm{TB} \rightarrow \mathrm{PB}}^{405}}{1+\mathrm{K}_{\mathrm{TB} \rightarrow \mathrm{PB}}^{405}}-\frac{\mathrm{K}_{\mathrm{TB} \rightarrow \mathrm{PB}}^{480}}{1+\mathrm{K}_{\mathrm{TB} \rightarrow \mathrm{PB}}^{480}}\right) \theta_{480}\left[\mathbf{S}_{\mathrm{tot}}\right]\left[1-\exp \left(-\frac{t}{\tau_{\mathrm{TB} \rightarrow \mathrm{PB}}}\right)\right] \\
+\frac{\left(Q_{\mathrm{PU}, 405}-Q_{\mathrm{TU}, 405}\right) I_{405}}{F_{405}(0)}\left(\frac{\mathrm{K}_{\mathrm{TU} \rightarrow \mathrm{PU}}^{405}}{1+\mathrm{K}_{\mathrm{TU} \rightarrow \mathrm{PU}}^{405}}-\frac{\mathrm{K}_{\mathrm{TU} \rightarrow \mathrm{PU}}^{480}}{1+\mathrm{K}_{\mathrm{TU} \rightarrow \mathrm{PU}}^{480}}\right)\left(1-\theta_{480}\right)\left[\mathbf{S}_{\mathrm{tot}}\right]\left[1-\exp \left(-\frac{t}{\tau_{\mathrm{TU} \rightarrow \mathrm{PU}}}\right)\right]
\end{array}
$$


where

$$
F_{405}(0)=\left(Q_{\mathrm{TB}, 405}\left[\mathbf{T B}_{480}^{\text {end }}\right]+Q_{\mathrm{TU}, 405}\left[\mathbf{T U}_{480}^{\text {end }}\right]+Q_{\mathrm{PB}, 405}\left[\mathbf{P B}_{480}^{\text {end }}\right]++Q_{\mathrm{PU}, 405}\left[\mathbf{P U}_{480}^{\text {end }}\right]\right) I_{405}
$$

Starting form $F_{405}(0)$, the fluorescence emission exponentially decays toward

$F_{405}($ end $)=\left[\left(\frac{Q_{\mathrm{TB}, 405}}{1+\mathrm{K}_{\mathrm{TB} \rightarrow \mathrm{PB}}^{405}}+\frac{\mathrm{K}_{\mathrm{TB} \rightarrow \mathrm{PB}}^{405} Q_{\mathrm{PB}, 405}}{1+\mathrm{K}_{\mathrm{TB} \rightarrow \mathrm{PB}}^{405}}\right) \theta_{480}+\left(\frac{Q_{\mathrm{TU}, 405}}{1+\mathrm{K}_{\mathrm{TU} \rightarrow \mathrm{PU}}^{405}}+\frac{\mathrm{K}_{\mathrm{TU} \rightarrow \mathrm{PU}}^{405} Q_{\mathrm{PU}, 405}}{1+\mathrm{K}_{\mathrm{TU} \rightarrow \mathrm{PU}}^{405}}\right)\left(1-\theta_{480}\right)\right]\left[\mathbf{S}_{\mathrm{tot}}\right] I_{405}$

Eq. 77 can be used to fit the fluorescence temporal evolution upon $405 \mathrm{~nm}$ illumination as a function of the analyte concentration. For a given $[\mathbf{L}]$, the method allows the extraction of the bound fraction $(\theta)$ based on the characteristic times and the amplitudes of photoisomerization at asymptotic $[\mathbf{L}]$. The simplified equation used for fitting is reported in Eqs. 4 and 8. Thus, the dependence of $\theta_{480}$ on $[\mathbf{L}]$ can be used to calibrate the rs-sensor (Hill equation Eq. 1). 


\section{References}

${ }^{1}$ Tsai-Wen Chen, Trevor J Wardill, Yi Sun, Stefan R Pulver, Sabine L Renninger, Amy Baohan, Eric R Schreiter, Rex A Kerr, Michael B Orger, Vivek Jayaraman, Loren L Looger, Karel Svoboda, and Douglas S Kim. Ultrasensitive fluorescent proteins for imaging neuronal activity. Nature, 499(7458):295-300, 2013.

${ }^{2}$ Vincent Gielen, Viola Mönkemöller, Yi Shen, Johan Hofkens, Pieter Vanden Berghe, Robert E Campbell, Benjamien Moeyaert, and Peter Dedecker. Absolute measurement of cellular activities using photochromic single-fluorophore biosensors. bioRxiv, 2020.

${ }^{3}$ Nordine Helassa, Borbala Podor, Alan Fine, and Katalin Török. Design and mechanistic insight into ultrafast calcium indicators for monitoring intracellular calcium dynamics. Scientific Reports, 6(1):38276, 2016.

${ }^{4}$ Luke M Oltrogge, Quan Wang, and Steven G Boxer. Ground-State Proton Transfer Kinetics in Green Fluorescent Protein. Biochemistry, 53(37):5947-5957, sep 2014.

${ }^{5}$ Xiaonan R Sun, Aleksandra Badura, Diego A Pacheco, Laura A Lynch, Eve R Schneider, Matthew P Taylor, Ian B Hogue, Lynn W Enquist, Mala Murthy, and Samuel S H. Wang. Fast GCaMPs for improved tracking of neuronal activity. Nature Communications, 4(1):2170, 2013.

${ }^{6}$ Lin Tian, S Andrew Hires, Tianyi Mao, Daniel Huber, M Eugenia Chiappe, Sreekanth H Chalasani, Leopoldo Petreanu, Jasper Akerboom, Sean A McKinney, Eric R Schreiter, Cornelia I Bargmann, Vivek Jayaraman, Karel Svoboda, and Loren L Looger. Imaging neural activity in worms, flies and mice with improved GCaMP calcium indicators. Nature Methods, 6(12):875-881, 2009.

${ }^{7}$ Pengcheng Wang, Jérôme Querard, Sylvie Maurin, Sarang S Nath, Thomas Le Saux, Arnaud Gautier, and Ludovic Jullien. Photochemical properties of Spinach and its use in selective imaging. Chem. Sci., 4(7):2865-2873, 2013. 